\title{
On the K-Theory of Cuntz-Krieger Algebras
}

\author{
By
}

\author{
David PASK ${ }^{*}$ and Iain RAEBURN **
}

\begin{abstract}
We extend the uniqueness and simplicity results of Cuntz and Krieger to the countably infinite case, under a row-finite condition on the matrix $A$. Then we present a new approach to calculat ing the $\mathrm{K}$-theory of the Cuntz-Krieger algebras, using the gauge action of $\mathrm{T}$, which also works when $A$ is a countably infinite $0-1$ matrix. This calculation uses a dual Pimsner-Voiculescu six-term exact sequence for algebras carrying an action of $\mathbf{T}$. Finally, we use these new results to calculate the $\mathrm{K}$-theory of the Doplicher-Roberts algebras.
\end{abstract}

\section{§1. Introduction}

In [4], [5], [3], Cuntz and Krieger studied the $C^{*}$-algebras generated by a family of $n$ non-zero partial isometries $S_{\imath}$, satisfying the Cuntz-Krieger relations

$$
S_{i}^{*} S_{k}=\delta_{i, k} \sum_{j=1}^{n} A(i, j) S_{j} S_{j}^{*}, 1 \leq i, k \leq n
$$

where $A$ is an $n \times n, 0-1$ matrix with no zero row or column. Let $\sum_{A}$ denote the set of finite sequences $\mu=\left(\mu_{1}, \cdots, \mu_{k}\right)$ with $1 \leq \mu_{\imath} \leq n$ and $A\left(\mu_{1}, \mu_{1+1}\right)=1$ for $i=1, \cdots, k-1$. The length $k$ of the sequnce $\mu$ is denoted by $|\mu|=k$. We may also think of $\mu \in \sum_{A}$ as a finite path in the infinite graph with vertices at each level labelled $1, \cdots, n$, in which for $k \geq 1$ there is an edge joining vertex $i$ on the $k^{\text {th }}$ level to vertex $j$ on the $k+1^{\text {st }}$ level if and only if $A(i, j) \neq 0$. For instance, the $k^{\text {th }}$ and $k+1^{\text {st }}$ levels of such a graph may look like the diagram given below:

Communicatde by H. Araki, July 10, 1995. Revised October 26, 1995.

1991 Mathematics Subject Classification (s) : 46L80

*.**Mathematics Department, University of Newcastle, NSW 2308, Australia. 


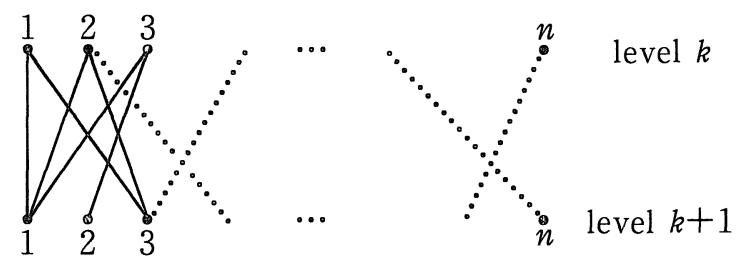

Definition 1.1. Let $\sum_{0}$ denote the set of $i_{0} . \quad 1 \leq i_{0} \leq n$ such that there are at least two different elements $\mu, \nu \in \sum_{A}$ such that $\mu_{1}=\nu_{1}=\mu_{|\mu|}=\nu_{|\nu|}=i_{0}$, and $\mu_{p}, \nu_{q}$ $\neq i_{0}$ for $1<p<|\mu|, 1<q<|\nu|$. The matrix $A$ satisfies condition (I) provided, for each $i$, with $1 \leq i \leq n$, there is some $m \geq 1$ and $i_{0} \in \sum_{0}$ with $A^{\mathrm{m}}\left(i, i_{0}\right) \neq 0$.

In particular, if $A$ is irreducible (i.e. for all $i, j$, there is a strictly positive integer $m=m(i, j)$ such that $\left.A^{\mathrm{m}}(i, j) \neq 0\right)$ and is not a permutation matrix, then $A$ satisfies condition (I). It was shown in [5] that when $A$ satisfies (I), the $C^{*}$-algebra generated by the $S_{i}, i=1, \cdots, n$ is independent of the choice of the partial isometries $S_{i}$, and simple whenever $A$ is irreducible. It may therefore be denoted by $\mathfrak{O}_{A}$. More precisely, in $[5,2.13$ and 2.14$]$ it was shown that:

Theorem 1.2. (i) Suppose that $A$ is a finite $\{0,1\}$ matrix satisfying (I) and $S_{i}, T_{i}, i=1, \cdots, n$, are two families of non-zero partial isometries satisfying the same Cuntz-Krieger relations (1). Then the map $S_{i} \mapsto T_{i}$ extends to an isomorphism of $C^{*}\left(S_{1}, \cdots, S_{n}\right)$ onto $C^{*}\left(T_{1}, \cdots, T_{n}\right)$.

(ii) If the matrix $A$ is irreducible and not a permutation matrix, then the $C^{*}$-algebra $\mathscr{O}_{A}=C^{*}\left(S_{1}, \cdots, S_{n}\right)$ is simple.

In the next section we give conditions $(\mathrm{J})$ on a countably infinite $0-1$ matrix $A$, under which the following theorem holds:

Theorem 1. (i) Suppose that $A$ is a countably infinite $\{0,1\}$ matrix satisfying $(\mathrm{J})$ and $S_{i}, T_{i}, i \in \mathbb{N}$ are two families of non-zero partial isometries satisfying the same infinite Cuntz-Krieger relations. Then the map $S_{i} \mapsto T_{\imath}$ extends to an isomorphism of $C^{*}\left(S_{i}\right)$ onto $C^{*}\left(T_{i}\right)$.

(ii) If the matrix $A$ row finite and irreducible then the $C^{*}$-algebre $\mathscr{O}_{\mathrm{A}}=C^{*}\left(S_{i}\right)$ is simple.

While condition $(\mathrm{J})$ is analogous to condition $(\mathrm{I})$, in order to get the simplicity result, we must assume irreducibility and a finiteness condition to ensure that certain topological obstacles do not occur. In the third section we review the proof of the following theorem of Kishimoto and Takai, [9, Theorem 2], since we shall need explicit details of the isomorphism later. 
Theorem 2. Let $B$ be unital $C^{*}$-algebra and $\beta$ a strongly continuous action of a compact group $G$ which has large spectral subspaces, then the fixed point algebra $B^{\beta}$ is stably isomorphic to $B \times{ }_{\beta} G$.

In the fourth section, we calculate the $\mathrm{K}$-theory of the $\mathscr{O}_{A}$ defined in the first section, generalising the results for finite matrices (see [5], [3] ). In particular, we prove the following:

Theorem 3. If $A$ is a countably infinite 0-1 matrix which satisfies condition ( $\mathrm{J})$ and is row finite, then there is an exact sequence

$$
0 \longrightarrow K_{1}\left(\mathscr{O}_{A}\right) \longrightarrow \tilde{Z}^{\infty} \stackrel{1-A^{t}}{\longrightarrow} \tilde{Z}^{\infty} \stackrel{i *}{\longrightarrow} K_{0}\left(\mathscr{O}_{A}\right) \longrightarrow 0
$$

so that $K_{1}\left(\mathscr{O}_{A}\right) \cong \operatorname{Ker}\left\{\left(1-A^{t}\right): \widetilde{\mathbb{Z}}^{\infty} \rightarrow \widetilde{\mathbf{Z}}^{\infty}\right\}$ and $K_{0}\left(\mathscr{O}_{A}\right) \cong \widetilde{\mathbf{Z}}^{\infty} / \operatorname{Im}\left(1-A^{t}\right) \widetilde{\mathbb{Z}}^{\infty}$. The map $i_{*}$ carries each canonical generator $\xi_{j}$ of $\widetilde{\mathbf{Z}}^{\infty}$ to the projection $\left[P_{j}\right]=$ $\left[S_{j} S_{j}^{*}\right]=\left[\begin{array}{ll}S_{j}^{*} & S_{j}\end{array}\right]$ in $K_{0}\left(\mathscr{O}_{A}\right)$ for each $j \in \mathbf{N}$. Thus we see that $K_{0}\left(\mathscr{O}_{A}\right)$ is generated by the projections $\left[P_{i}\right]$, for each $i \in \mathbf{N}$, subject to $\left[P_{i}\right]=\sum_{j=1}^{\infty} A(i, j)$ $\left[P_{j}\right]$ induced from the Cuntz-Krieger relations.

While this result is not suprising, and could conceivably be deduced from the known results finite $A$, we feel our proof is of some interest even in the finite case. We bypass the natural realisation of $\mathscr{O}_{A} \otimes \mathcal{K}$ as a crossed product by $\mathbb{Z}$, using instead the gauge action of $\mathbf{T}$ on $\mathscr{O}_{A}$, and the six-term exact sequence in $\mathrm{K}$-theory dual to the Pimsner-Voiculescu of $[11, \S 3]$ (which curiously was described in $[1, \S 10.6]$ as of limited use). Thus our argument give an alternative approach to the calculation of $K *\left(\mathscr{O}_{A}\right)$ which may be slightly more accessible.

Our original motivation for this work was to calculate the $\mathrm{K}$-theory of the algebras $\mathscr{O}_{\rho}$ appearing in the Doplicher-Roberts duality theory for compact groups. In [10], it was shown that $\mathscr{O}_{\rho}$ was isomorphic to a corner in a $C^{*}$-algebra generated by an infinite Cuntz-Krieger family; now we know by Theorem 1 that this Cuntz-Krieger algebra $\mathscr{O}_{A_{\rho}}$ is simple, we have $K_{*}\left(\mathscr{O}_{\rho}\right) \cong K_{*}$ $\left(\mathscr{O}_{A_{\rho}}\right)$, and we can use Theorem 3 to compute $K_{*}\left(\mathscr{O}_{\rho}\right)$. In fact, we can do better: we can identify $\widetilde{\mathbb{Z}}^{\infty}$ with the representation ring $\mathscr{R}(G)$, and $K_{*}\left(\mathscr{O}_{\rho}\right)$ with the kernel and cokernel of the map $[\pi] \mapsto[\pi \otimes \rho]$ on $\mathscr{R}(G)$. In the fifth section we shall briefly discuss this approach.

\section{Acknowledgement}

We originally learned about Doplicher-Roberts duality and the computation of $K *\left(\mathscr{O}_{\rho}\right)$ in seminars, discussions and joint work with Colin Sutherland (cf. [10], [12]). We thank him for his help and interest, and particularly for suggesting to us that we should interpret our results in terms of the representation 
ring.

This research was supported by the Australian Research Council.

\section{\$2. Infinite Cuntz-Krieger Algebras}

\subsection{Uniqueness and Simplicity}

The following definitions and results are extensions of those given in $[5$, p.253], where it is claimed that their results for finite matrices carry over to the infinite case. Upon closer inspection, this does not seem as straightforward as originally thought. Given a countably infinite $0-1$ matrix, $A$, with no zero row or column, we consider the $C^{*}$-algebra generated by non-zero partial isometries $S_{\imath}, i \in \mathbb{N}$, satisfying the Cuntz-Krieger relations,

$$
S_{i}^{*} S_{k}=\delta_{i, k} \sum_{j=1}^{\infty} A(i, j) S_{j} S_{j}^{*}, \quad \text { for all } i, k \in \mathbb{N}
$$

where the infinite sum above converges in the strong operator topology. If we have $\mu=\left(\mu_{1}, \cdots, \mu_{k}\right) \in \Sigma_{A}$, we write $S_{\mu}=S_{\mu_{1}} \cdots, S_{\mu_{k}}$, and then each $S_{\mu}$ is a partial isometry with range projection denoted by $P_{\mu}=S_{\mu} S_{\mu}^{*}$. In particular, $P_{i}=S_{\imath} S_{i}^{*}$ denotes the range projection of each partial isometry $S_{\imath}$, for all $i \in$ $\mathbb{N}$.

Definition 2.1.1. Let $\sum_{\infty}$ denote the set of $i_{\infty} \in \mathbb{N}$ such that there are at least two distinct paths $\mu, \nu \in \sum_{A}$, such that $\mu_{1}=\nu_{1}=\mu_{|\mu|}=\nu_{|\nu|}=i_{\infty}$, and $\mu_{p}, \nu_{q} \neq i_{\infty}$ for $1<p<|\mu|, 1<q<|\nu|$. The matrix $A$ satisfies condition (J) provided there is a finite subeset $\Sigma_{1} \subseteq \sum_{\infty}$ such that for each $i \in \mathbb{N}$ there is some $m \geq 1$ and $i_{1} \in \Sigma_{1}$ with $A^{m}\left(i, i_{1}\right) \neq 0$.

Note 2.1.2. As in $[5, \mathrm{p} .254]$ we note that if the countably infinite $0-1 \mathrm{ma}$ $\operatorname{trix} A$ is irreducible, then it satisfies condition $(\mathrm{J})$, because irreducibility implies that there is at least one vertex with at least two edges eminating from it, from which we may construct the required paths $\mu, \nu$. Here we can dispense with the requirement for $A$ not to be a permutation matrix as the above construction relies on the infinite nature of the graph as well as the irreducibility of the matrix $A$.

Also, we note that, for countably infinite $0-1$ matrices, condition $(\mathrm{J})$ is stronger than the full countably infinite version of condition (I) where we do not demand the existence of the finite subset $\Sigma_{1}$. 
Example 2.1.3. Consider the matrices

$$
A_{1}=\left(\begin{array}{ccccc}
0 & 1 & 0 & 0 & \cdots \\
1 & 1 & 0 & 0 & \cdots \\
0 & 1 & 1 & 0 & \cdots \\
0 & 0 & 1 & 1 & \cdots \\
\vdots & \vdots & \vdots & \vdots & \ddots
\end{array}\right) \quad A_{2}=\left(\begin{array}{ccccc}
0 & 1 & 0 & 0 & \cdots \\
1 & 1 & 0 & 0 & \cdots \\
0 & 0 & 0 & 1 & \cdots \\
0 & 0 & 1 & 1 & \cdots \\
\vdots & \vdots & \vdots & \vdots & \ddots
\end{array}\right) A_{3}=\left(\begin{array}{ccccc}
0 & 1 & 0 & 0 & \cdots \\
1 & 1 & 1 & 0 & \cdots \\
0 & 1 & 1 & 1 & \cdots \\
0 & 0 & 1 & 1 & \cdots \\
\vdots & \vdots & \vdots & \vdots & \ddots
\end{array}\right) .
$$

Then $A_{1}$ satisfies condition $(\mathrm{J})$, with $\sum_{\infty}=\sum_{1}=\{1,2\}$. On the other hand $A_{2}$ satisfies the full countably infinite version of condition (I), but does not satisfy condition $(\mathrm{J})$, because $\sum_{\infty}=\mathbf{N}$, and each $i_{\infty} \in \sum_{\infty}$ is connected to precisely two indices. The irreducible matrix $A_{3}$ satisfies condition $(\mathrm{J})$, even though $\Sigma_{\infty}=$ $\mathbf{N}$; we can take any finite subset for $\Sigma_{1}$.

We shall henceforth assume that our infinite 0-1 matrix $A$ satisfies condition $(\mathrm{J})$.

Lemma 2.1.4. For each $n \in \mathbf{N}$, there is a positive integer $m_{n}$ and a partition $I_{n}^{k}, 1 \leq k \leq m_{n}$ of $\mathbf{N}$ such that:

(i) $I_{n}^{k}=\{k\}$ if $1 \leq k \leq n$.

(ii) For $1 \leq i \leq n$, there are subsets $K_{i}$ of $\left\{1, \cdots, m_{n}\right\}$ such that for all $j \in \mathbf{N}$

$$
A(i, j)=1 \text { if and only if } j \in \bigcup_{k \in K_{t}} I_{n}^{k}
$$

Proof. Add to $I_{n}^{k}=\{k\}, 1 \leq k \leq n$, the partition of $\{n+1, n+2, \cdots$,$\} generated$ by the sets

$$
J_{i}=\{j>n: A(i, j)=1\}
$$

for $i=1, \cdots, n$ and the set $\mathbb{N} \backslash\left(\cup_{i=1}^{n} J_{\imath}\right)$, giving $m_{n}$ sets in all. Roughly speaking, $I_{n}^{k}$, for $k>n$ represent the vertices which can be reached in one step from each of some, possibly the subsets of $\{1, \cdots, n\}$. The sets $K_{\imath}, i=1, \cdots, n$ consist of those superscripts $k$ of the subsets $I_{n}^{k}$ whose vertices are reached from vertex $i$ in exactly one step. We may see that each $K_{i}$ is non-empty from the definition of the $J_{i}$. Finally, the number $m_{n}$ is finite for each $n$, since the number of disjoint subsets of $\mathbf{N}$ which the $J_{i}, i=1, \cdots, n$ can generate is finite. 
Definition 2.1.5. For each $n \in \mathbb{N}$ define an $m_{n} \times m_{n} 0-1$ matrix $B_{n}$ by

$$
B_{n}(k, l)=\left\{\begin{array}{l}
1 \text { if } A(i, j)=1 \text { for some } i \in I_{n}^{k}, j \in I_{n}^{k} \\
0 \text { otherwise. }
\end{array}\right.
$$

We think of the new vertices $\left\{1, \cdots, m_{n}\right\}$ as those obtained by identifying all vertices comprising $I_{n}^{k}$, and joining new vertex $k$ to new vertex $l$ if any vertex in $I_{n}^{k}$ is joined to any vertex from $I_{n}^{l}$ in the original graph. Note that, by construction, no row or column of $B_{n}$ is zero.

\section{Lemma 2.1.6.}

(i) If $n$ is large enough, then the matrix $B_{n}$ satisfies condition (I).

(ii) If $1 \leq k \leq n$, then $K_{k}=\left\{l: B_{n}(k, l)=1\right\}$.

Proof. From 2.1.1, let $\Sigma_{1} \subset \mathbf{N}$ be given for the countably infinite $0-1$ matrix $A$. Choose $n$ sufficiently large so that $n \geq i_{1}$ for each $i_{1} \in \Sigma_{1}$ and each of the designated paths $\mu, \nu \in \sum_{A}$ for $i_{1}$ only visit the first $n$ vertices. This means that for this $n$, we have $i_{1} \in \sum_{0}$ for $B_{n}$ since we are not identifying any edges used in the paths $\mu, \nu$. Hence the set $\Sigma_{0}$ for the matrix $B_{n}$ contains $\Sigma_{1}$. We know from condition $(\mathrm{J})$, that all vertices in the original graph connect to a vertex in $\Sigma_{1}$. Thus, since in constructing $B_{n}$ we are effectively adding new paths to the original graph, each of the new vertices must connect to a vertex in $\Sigma_{1} \subseteq$ $\Sigma_{0}$, which is sufficient for condition (I) (see 1.1).

For (ii), if $k \leq n$, then by definition, $B_{n}(k, l)=1$ if and only if there is some $j \in I_{n}^{l}$ such that $\mathrm{A}(k, j)=1$. Hence this is so if and only if

$$
I_{n}^{l} \subset \bigcup_{m \in K_{k}} I_{n}^{m}
$$

that is, if and only if $l \in K_{k}$.

Example 2.1.7. For the matrices $A_{1}, A_{2}$ and $A_{3}$ given in 2.1.3, we see how the above proof gives rise to the need for condition ( $\mathrm{J}$ ) over the full infinite version of condition (I). For $A_{1}$ if we choose $n=2$, then we have

$$
B_{2}=\left(\begin{array}{lll}
0 & 1 & 0 \\
1 & 1 & 0 \\
0 & 1 & 1
\end{array}\right)
$$


which satisfies condition (I). However, for $A_{2}$, whenever $n \geq 2$, say 2 , then we have

$$
B_{2}=\left(\begin{array}{lll}
0 & 1 & 0 \\
1 & 1 & 0 \\
0 & 0 & 1
\end{array}\right)
$$

which does not satisfy condition (I), as the third vertex only connects to itself. For matrix $A_{3}$, whenever $n \geq 2$, say 2 , we obtain

$$
B_{2}=\left(\begin{array}{llll}
0 & 1 & 0 & 0 \\
1 & 1 & 1 & 0 \\
0 & 1 & 1 & 1 \\
0 & 0 & 1 & 1
\end{array}\right)
$$

which does satisfy condition (I).

Theorem 2.1.8. Suppose that $A$ is a countably infinite $0-1$ matrix satisfying condition $(\mathrm{J})$ and that $\left\{S_{i}\right\},\left\{T_{i}\right\}$ are two families of non-zero partial isometries satisfying the same Cuntz-Krieger relations (2). Then there is an isomorphism $\phi$ of $C^{*}\left(S_{i}\right)$ onto $C^{*}\left(T_{i}\right)$ such that $\phi\left(S_{i}\right)=T_{i}$ for all $i \in \mathbf{N}$.

Proof. Define $S_{k, n}=S_{k}$, for $1 \leq k \leq n$, and for $n<k \leq m_{n}$ take $S_{k, n}$ to be a partial isometry with range projection

$$
S_{k, n} S_{k, n}^{*}=\sum_{j \in I_{n}^{k}}^{\text {s.o. }} S_{j} S_{j}^{*}
$$

and initial projection

$$
S_{k, n}^{*} S_{k, n}=\sum_{l=1}^{m n} B_{n}(k, l)\left(\sum_{j \in I_{n}^{l}}^{\text {s.o. }} S_{j} S_{j}^{*}\right) .
$$

Since the inside sum is over a finite set, the right hand side converges in the strong operator topology, as do all the sums which follow. With the above definitions, we claim that the partial isometries $S_{k, n}, 1 \leq k \leq m_{n}$ satisfy

$$
S_{k, n}^{*} S_{k, n}=\sum_{l=1}^{m_{n}} B_{n}(k, l) S_{l, n} S_{l, n}^{*}
$$


To see this, for $1 \leq k \leq n$, we have that

$$
\begin{aligned}
S_{k, n}^{*} S_{k, n}=S_{k}^{*} S_{k} & =\sum_{j \in J_{k}} A(k, j) S_{j} S_{j}^{*} \\
& =\sum_{j \in \cup \cup \in K_{k} I_{n}^{l}} S_{j} S_{j}^{*} \text { by 2.1.4ii) } \\
& =\sum_{l \in K_{k}}\left(\sum_{j \in I_{n}^{l}} S_{j} S_{j}^{*}\right) \\
& \left.=\sum_{l=1}^{m_{n}} B_{n}(k, l)\left(\sum_{j \in I_{n}^{l}} S_{j} S_{j}^{*}\right) \quad \text { by } 2.1 .6 \mathrm{ii}\right) \\
& =\sum_{l=1}^{m_{n}} B_{n}(k, l) S_{l, n} S_{l, n}^{*} \text { by (3). }
\end{aligned}
$$

If $k>n$ then the condition holds by definition of the $S_{k, n}$.

If we do the same construction for the $T_{i}$ 's; then, since by 2.1.6 i) $B_{n}$ satisfies condition (I) we may apply the Cuntz-Krieger theorem 1.2 i) to give an isomorphism of $C^{*}\left(S_{1, n}, \cdots, S_{m_{n}, n}\right)$ onto $C^{*}\left(T_{1, n}, \cdots, T_{m_{n}, n}\right)$ carrying $S_{k, n}$ to $T_{k, n}$ for $1 \leq k \leq m_{n}$. This restricts to an isomorphism $\phi_{n}$ of $C^{*}\left(S_{1}, \cdots, S_{n}\right)$ onto $C^{*}\left(T_{1}, \cdots, T_{n}\right)$, and hence we have a countable family of isomorphisms $\phi_{n}$ defining an isomorphism

$$
\phi: \bigcup_{n} C^{*}\left(S_{1}, \cdots, S_{n}\right) \rightarrow \bigcup_{n} C^{*}\left(T_{1}, \cdots, T_{n}\right)
$$

Each $\phi_{n}$ is isometric, hence so is $\phi$, and $\phi$ extends to the closure and has the desired properties.

Corollary 2.1.9. If $A$ is a countably infinite matrix which satisfies condition $(\mathrm{J})$, then there is an action $\alpha$ (called the gauge action) of $\mathbb{T}$ on $\mathfrak{O}_{A}$ such that

$$
\alpha_{z}\left(S_{i}\right)=z S_{i} \text { for all } i \in \mathbb{N} \text {. }
$$

Proof. To see this, we note that the partial isometries $T_{i}=z S_{\imath}, i \in \mathbb{N}$ also satisfy condition ( $\mathrm{J}$ ) and the infinite Cuntz-Krieger relations (2). Thus Theorem 2.1.8 gives an isomorphism $\alpha_{z}$ of $\mathscr{O}_{A}$. The map $z \mapsto \alpha_{z}(a)$ is continuous when $a$ lies in the ${ }^{*}$-subalgebra generated by the $S_{i}$ and hence for all $a$ $\in \mathscr{O}_{A}$. 
Definition 2.1.10. The countably infinite $0-1$ matrix $A$ is said to be row-finite if, for each $i \in \mathbf{N}$ the number of $j \in \mathbf{N}$ with $A(i, j) \neq 0$ is finite.

Note 2.1.11. The row-finite condition defined above implies that the sums occurring in the infinite Cuntz-Krieger relations (2) are finite, and hence trivially converge. Also, from [15, Lemma 1.5] the row-finite condition on $A$ is a necessary and sufficient condition for the one-sided infinite path space $X_{A}^{+}$to be given a locally compact topology. Irreducibility then implies that this topology has no isolated points, cf. [5, p.254].

Corollary 2.1.12. Suppose the countably infinite 0-1 matrix $A$ is row-finite and irreducible, then the $C^{*}$-algebra $\mathscr{O}_{A}=C^{*}\left(S_{\imath}\right)$ is simple.

Proof. Suppose $I \triangleleft \mathscr{O}_{A}$ is a proper closed 2 -sided $*$-ideal. We claim that no $S_{\imath}$ can belong to $I$. For if $S_{\imath} \in I$ for some $i \in \mathbb{N}$, then $S_{\imath}{ }^{*} S_{\imath} \in I$, in which case $S_{j}=S_{i}^{*} S_{i} S_{j}$ belongs to $I$ whenever $A(i, j)=1$. By induction we would then have $S_{\mu} \in I$ whenever $\mu \in \sum_{A}$ satisfied $\mu_{1}=i$ or $A\left(i, \mu_{1}\right)=1$. Since $A$ is irreducible by hypothesis, we may thus show that $S_{k} \in I$ for all $k \in \mathbf{N}$ since $S_{k}=$ $S_{\mu}^{*} S_{\mu} S_{k} \in I$ for any path $\mu \in \sum_{A}$ with $A\left(\mu_{|\mu|}, k\right)=1$ and $\mu_{1}=i$ say. Since we know that $\mathscr{O}_{A}=C^{*}\left(S_{\imath}\right)$, this would imply that $I=\mathscr{O}_{A}$, which is a contradiction.

Since $S_{\imath} \notin I$, for all $i \in \mathbf{N}$ and the sums in (2) are finite, the images $q\left(S_{i}\right)=S_{i}+I$ in the quotient algebra $\mathscr{O}_{A} / I$ are non-zero partial isometries satisfying the infinite Cuntz-Krieger relations (2). Thus there is an isomorphism $\phi$ of $\mathscr{O}_{A} / I$ onto $\mathscr{O}_{A}$, such that $\phi\left(S_{\imath}+I\right)=S_{\imath}$ for all $i \in \mathbb{N}$. But then the composition $\phi \circ q$ must be the identity, so $I=\{0\}$, as required.

Remark 2.1.13. (i) This last argument will not work if $A$ is not row-finite, since the quotient map need not respect the infinite Cuntz-Krieger relations (2), because they involve strong operator convergence.

(ii) For the algebra $\mathscr{O}_{\infty}$, the countably infinite $0-1$ matrix $A$ consists entirely of 1's, and it may be considered as the direct limit of the $\mathscr{O}_{\mathrm{B} n}$. Thus we can immediately deduce the simplicity of $\mathscr{O}_{\infty}$ and compute its $\mathrm{K}$-theory (using the continuity of $\mathrm{K}$-theory). In general, our proof of Theorem 2.1.8 does not show how to compute $K_{0}\left(\mathscr{O}_{A}\right)$, because the algebras $\mathscr{O}_{B n}$ lie partly outside $\mathscr{O}_{A}$.

\subsection{The $\mathrm{AF}$-core}

Throughout this section $A$ will be row-finite, for such $A$, as in $[5$, Lemma 2.2], every word in $S_{\imath}$ and $S_{\imath}^{*}$ is a linear combination of terms of the form $S_{\mu} P_{j}$ $S_{\nu}^{*}$ for some $j \in \mathbb{N}$ and $\mu, \nu \in \sum_{A}$. Following [5, p.253], for each $i \in \mathbb{N}$ and $k \geq$ 0 , we let $\mathscr{F}_{k}(\mathrm{i})$ be the $C^{*}$-algebra generated by all elements of the form $E_{\mu, \nu}^{\imath}=S_{\mu} P_{\imath} S_{\nu}^{*}$ where $|\mu|=|\nu|=k$. Since $A$ is row finite, $\mathscr{F}_{k}(i)$ is a finite 
dimensional full matrix algebra, since there can only be finitely many paths in $\sum_{A}$ of length $k$ ending at vertex $i$.

Definition 2.2.1. For $j \in \mathbf{N}, k \geq 0$, let $V_{k}^{j}=\left\{i \in \mathbf{N}: A^{k}(h, i) \neq 0\right.$ for some 1 $\leq h \leq j\}$, and for $i \in V_{k}^{j}$ let $\mathscr{F}_{k}^{j}(i)$ be the $C^{*}$-algebra generated by all elements of the form $E_{\mu, \nu}^{i}$ where $|\mu|=|\nu|=k$, and $1 \leq \mu_{1}, \nu_{1} \leq j$. We denote by $\mathscr{F}_{A}$ the closure of the infinite union $\cup_{k, j} \cup_{i \in V_{k}^{\prime}} \mathscr{F}_{k}^{j}(i)$.

Lemma 2.2.2. For each $j \in \mathbb{N}, k \geq 0$ and $i \in V_{k}^{j}$ the $C^{*}$-algebra $\mathscr{F}_{k}^{j}(i)$ is a full matrix algebra with matrix units $E_{\mu, \nu}^{i}$, where $|\mu|=|\nu|, 1 \leq \mu_{1}, \nu_{1} \leq j$ and $\mu, \nu$ $\in \sum_{A}$. The $C^{*}$-algebra $\mathscr{F}_{A}$ is an $A F$-algebra.

Proof. As in [5, Proposition 2.3] the elements $E_{\mu, \nu}^{i}$, with $|\mu|=|\nu|=k$ satisfy

$$
E_{\mu, \nu}^{i} E_{\varkappa, \sigma}^{j}=\delta_{i, j} \delta_{\nu, \varkappa} E_{\mu, \sigma}^{i}
$$

for $\mu, \nu, \kappa, \sigma \in \sum_{A}$. This implies in particular that, for fixed $i$, the $E_{\mu, \nu}^{i}$ form a system of matrix units; since $A$ is row-finite there are only finitely many paths $\mu \in \sum_{A}$ with $1 \leq \mu_{1} \leq j$ and $\mu_{k}=i$, hence $\mathscr{F}_{k}^{j}(i)$ is a full matrix algebra.

From the definition we know that

$$
\mathscr{F}_{A}=\overline{\operatorname{span}}\left\{S_{\mu} P_{i} S_{\nu}^{*}:|\mu|=|\nu|=k, A\left(\mu_{k}, i\right)=A\left(\nu_{k}, i\right)=1\right\} .
$$

We order these elements first by fixing the level $k$ at which we operate, then by fixing the terminating vertex at level $k$ and finally by restricting the starting points of our paths $\mu, \nu \in \Sigma_{A}$ to a range $1 \leq \mu_{1}, \nu_{1} \leq j$. As $j$ increases, we just add more matrix units $E_{\mu, \nu}^{i}$ to the collection spanning $\mathscr{F}_{k}^{j}(i)$. Thus the increasing union of matrix algebras, $\mathscr{F}_{k}(i)=\cup_{j} \mathscr{F}_{k}^{j}(i)$ is either itself a matrix algebra, or a copy of the compacts.

The algebras $\mathscr{F}_{k}(i)$, for $i \in \mathbb{N}$ are mutually orthogonal by (4), so $\mathscr{F}_{k}=U_{i}$. $\mathscr{F}_{k}(i)$ is actually a $C^{*}$-algebraic direct sum $\bigoplus_{i} \mathscr{F}_{k}(i)$ of $C^{*}$-algebras isomorphic to $\mathscr{K}(\mathscr{H})$ for some, possibly finite dimensional Hilbert space. In particular, each $\mathscr{F}_{k}$ is an AF algebra, and hence so is $\mathscr{F}_{A}$, which completes the proof.

Lemma 2.2.3. With the above notation, we have that $\mathscr{F}_{A}=\mathscr{O}_{A}^{\alpha}$.

Proof. For $a \in \mathscr{O}_{A}$ the operator $P_{\alpha}(a)=\int_{\mathbb{T}} \alpha_{z}(a) d z$ is a conditional expectation of $\mathscr{O}_{A}$ onto $\mathcal{O}_{A}^{\alpha}$ of norm 1. By definition of $\alpha$, each $S_{\mu} P_{i} S_{\nu}^{*}$ with $|\mu|$ $=|\nu|$ lies in $\mathscr{O}_{A}^{\alpha}$, hence $\mathscr{F}_{k}^{j}(i) \subset \mathscr{O}_{A}^{\alpha}$ for all $j \in \mathbb{N}, k \geq 0, i \in V_{k}^{j}$. Thus by 2.2 .2 
we have that $\mathscr{F}_{A} \subseteq \mathscr{O}_{A}^{\alpha}$.

Any $a \in \mathscr{O}_{A}$ may be approximated by finite linear combinations of $\left\{S_{\mu} S_{\nu}^{*}\right.$ : $\left.\mu, \nu \in \sum_{A}\right\}$. From the definition of $P_{\alpha}$ we see that

$$
P_{\alpha}\left(S_{\mu} S_{\nu}^{*}\right)=\int_{\mathbf{T}} z^{|\mu|-|\nu|} S_{\mu} S_{\nu}^{*} d z
$$

which is non-zero if and only if $|\mu|=|\nu|$. So, if $a=P_{\alpha}(a) \in \mathscr{O}_{A}^{\alpha}$, continuity of $P_{\alpha}$ implies that $a$ can be approximated by linear combinations of $\left\{S_{\mu} S_{\nu}^{*}: \mu, \nu\right.$ $\left.\in \sum_{A},|\mu|=|\nu|\right\}$. But each linear combination belongs to $\mathscr{F}_{A}$, which gives us that $\mathscr{O}_{A}^{\alpha} \supseteq \mathscr{F}_{A}$, and completes the proof.

\section{§3. A Result of Kishimoto and Takai}

\subsection{General Theory}

Convention 3.1.1. Throughout this section, $B$ will be a unital $C^{*}$ algebra with identity 1 , the identity map on $B$ will be denoted by $i, G$ a compact abelian group with normalised Haar measure, and discrete dual group $\bar{G}$. $\mathscr{H}$ will denote an infinite dimensional separable Hilbert space, and $\mathscr{K}$ the $C^{*}$ algebra of compact operators on $\mathscr{H}$, generated by matrix units $e_{i j}, i, j \in \mathbf{N}$. The compact operators on the Hilbert space $L^{2}(G)$ will be denoted $\mathscr{K}\left(L^{2}(G)\right.$ ). We shall use the following definition which is to be found in [11, §2]:

Definition 3.1.2. Let $\beta$ be a (strongly continuous) action of a compact abelian group $G$ on a $C^{*}$-algebra $B$, and $B^{\beta}$ its fixed point algebra. For a character $\chi$ $\in \widehat{G}$, we let $B^{\beta}(\chi)$ denote the spectral subspace $\left\{b \in B: \beta_{t}(b)=\chi(t) b\right.$ for all $t \in$ $G\}$. We say that $\beta$ has large spectral subspaces if $\overline{B^{\beta}(\chi)^{*} B^{\beta}(\chi)}=B^{\beta}$ for each $\chi$ $\in \bar{G}$

Definition 3.1.3. Let $\lambda, \rho$ denote the left, right regular representations of a compact group $G$ on $L^{2}(G)$, i.e.

$$
\left(\lambda_{s} \xi\right)(t)=\xi\left(s^{-1} t\right) \text { and }\left(\rho_{s} \xi\right)(t)=\xi(t s)
$$

for $s, t \in G$ and all $\xi \in L^{2}(G)$. Also, let $M$ denote the representation of $C_{o}(G)$ as multiplication operators on $L^{2}(G)$ given, for $f \in C_{o}(G)$, by

$$
\left(M_{f} \xi\right)(s)=(f \xi)(s) \text { for } \xi \in L^{2}(G) .
$$

Let $\tau$ denote the action of $G$ on $C_{0}(G)$ by left translation, that is $\tau_{s}(f)(t)=f(s t)$ 
for $f \in C_{0}(G), s, t \in G$.

Here we shall consider the group $C^{*}$-algebra $C^{*}(G)$ to be the closed linear span of $\{\lambda(\gamma): \gamma \in \widehat{G}\}$ in $\mathscr{L}\left(L^{2}(G)\right)$, where $\lambda(\gamma)$ is the operator given by $\int_{G} \gamma(s) \lambda_{s}$ $d s$ (this differs from the definition used in [9, p. 387], we believe that this version is more common). Finally, let Ad $\rho$ denote the adjoint action of $G=\widehat{\widehat{G}}$ on $\mathscr{K}\left(L^{2}(G)\right)$, given, for $T \in \mathscr{K}\left(L^{2}(G)\right)$ and $s \in G$ by $A d \rho_{s}(T)=\rho_{s} T \rho_{s}^{*}$.

Lemma 3.1.4. The algebra $\mathscr{K}\left(L^{2}(G)\right)$ is the closed span of $\left\{M_{\chi} \lambda(\gamma): \chi\right.$, $\gamma \in \widehat{G}\}$; note that

$$
\lambda(\gamma) M_{\chi}=M_{x} \lambda(\bar{\chi} \gamma)
$$

The spectral subspaces of the action $A d \rho$ on $\mathscr{K}\left(L^{2}(G)\right)$ are given by

$$
\mathscr{K}\left(L^{2}(G)\right) \operatorname{Ad\rho }(\chi)=M_{\chi} \lambda\left(C^{*}(G)\right)
$$

for all $\chi \in \widehat{G}$.

Proof. We note, from $\left[14\right.$, Example 4] that the triple $\left(\mathscr{K}\left(L^{2}(G)\right), M, \lambda\right)$ is a crossed product for $\left(C_{0}(G), G, \tau\right)$. Thus from [14, p.322] we know that

$$
\overline{\operatorname{span}}\left\{M_{f} \lambda(z): f \in C_{0}(G), z \in C_{\mathrm{C}}(G)\right\}=\mathscr{K}\left(L^{2}(G)\right)
$$

and by Stone-Weierstrass we also know that the closed span of $\widehat{G}$ is dense in $C_{0}(G)$ and $C_{\mathrm{c}}(G)$. Hence the operators $M_{\chi} \lambda(\gamma)$ span a dense subspace of $\mathscr{K}\left(L^{2}(G)\right)$. Equation (5) is an easy calculation. For the last part, note that $\rho_{s}$ commutes with $\lambda_{t}$ for $s, t \in G$, and hence with $\lambda\left(C^{*}(G)\right)$, and

$$
\operatorname{Ad} \rho_{s}\left(M_{\chi}\right)=\chi(s) M_{\chi}
$$

so $M_{\chi} \lambda\left(C^{*}(G)\right)$ is certainly contained in the spectral subspace. On the other hand the projection $P_{\chi}$ onto $\mathscr{K}\left(L^{2}(G)\right)^{\text {Ado }}(\chi)$ is given by

$$
P_{\chi}(T)=\int_{G} A d \rho_{t}(T) \overline{\chi(t)} d t
$$

and hence

$$
P_{\chi}\left(M_{\varkappa} \lambda(\gamma)\right)= \begin{cases}M_{\varkappa} \lambda(\gamma) & \text { if } \kappa=\chi \\ 0 & \text { otherwise }\end{cases}
$$

Since the $M_{\varkappa} \lambda(\gamma)$ span a dense subspace of $\mathscr{K}\left(L^{2}(G)\right)$, and $M_{\chi} \lambda\left(C^{*}(G)\right)$ is 
closed, it follows that $\mathscr{K}\left(L^{2}(G)^{A d \rho}(\chi) \subseteq M_{\chi} \lambda\left(C^{*}(G)\right)\right.$, which completes the proof.

\subsection{The Main Theorem}

Lemma 3.2.1. Let $\beta: G \rightarrow A u t B$ be a (strongly continuous) action of a compact abelian group $G$. Then there is an isomorphism $\Phi$ of the crossed product $B \times_{\beta} G$ onto $\left(B \otimes \mathscr{K}\left(L^{2}(G)\right)\right)^{\beta}{ }^{\otimes}$ Ado , such that

$$
\Phi\left(i_{B}(b) i_{G}(\gamma)\right)=b \otimes M_{\bar{\chi}} \lambda(\gamma)
$$

where $\gamma, \chi \in \widehat{G}, b \in B^{\beta}(\chi)$.

Proof. Define a map $\beta^{-1}: B \rightarrow C_{b}(G, B) \subset M\left(B \otimes C_{0}(G)\right)$ by the formula $\beta^{-1}(b)(t)=\beta_{t^{-1}}(b)$. Now let

$$
j_{B}=(i \otimes M) \circ \beta^{-1}: B \rightarrow M\left(B \otimes \mathscr{K}\left(L^{2}(G)\right)\right)
$$

and let

$$
j_{G}=1 \otimes \lambda: G \rightarrow U M\left(B \otimes \mathscr{K}\left(L^{2}(G)\right)\right) .
$$

A straightforward calculation, as at the top of $[14, \mathrm{p} .326]$, shows that $\left(j_{B}\right.$, $\left.j_{G}\right)$ is covariant on $(B, G, \beta)$. Then, [14, Proposition $\left.2(2)\right]$ gives us a non-degenerate homomorphism $\Phi=j_{B} \times j_{G}$ of $B \times{ }_{\beta} G$ into $M\left(B \otimes \mathscr{K}\left(L^{2}(G)\right)\right)$, such that $\Phi \circ i_{B}=j_{B}$ and $\Phi \circ i_{G}=j_{G}$. For $b \in B^{\beta}(\chi), \chi, \in \widehat{G}$, we have $\beta^{-1}(b)=$ $b \otimes \bar{\chi}$, and an easy calculation gives (7). It follows that $\Phi$ takes values in $B \otimes$ $\mathscr{K}\left(L^{2}(G)\right)$ and another calculation using (6) shows that the image is fixed under $\beta \otimes A d \rho$.

Since $\mathscr{K}\left(L^{2}(G)\right)=\overline{\operatorname{span}}\left\{M_{\chi} \lambda(\gamma): \chi, \gamma \in \widehat{G}\right\},\left(B \otimes \mathscr{K}\left(L^{2}(G)\right)\right)^{\beta} \otimes_{A d \rho}$ is spanned by elements of the form

$$
\int_{G} \beta_{s} \otimes A d \rho_{s}\left(c \otimes M_{\bar{\chi}} \lambda(\gamma)\right) d s=\int_{G} \overline{\chi(s)} \beta_{s}(c) d s \otimes M_{\bar{\chi}} \lambda(\gamma) d s
$$

where $c \in B$ and $\gamma, \chi \in \widehat{G}$. But $b=\int_{G} \overline{\chi(s)} \beta_{s}(c) d s$ lies in $B^{\beta}(\chi)$, so this shows that $\Phi$ has dense range and hence maps $B \times{ }_{\beta} G$ onto $\left(B \otimes \mathcal{K}\left(L^{2}(G)\right)\right)^{\beta} \otimes_{A d o}$.

Finally, we note that since $\Phi$ is the regular representation of $B \times{ }_{\beta} G$ induced from $i: B \rightarrow B$, and $G$ is amenable, we have that $\Phi$ is a faithful representation of $B \times{ }_{\beta} G$ (see $\left.[13,7.7 .8]\right)$. Thus $\Phi$ is injective, and this completes our proof. 
Let $c$ denote the trivial action of $G$ on $\mathscr{K}$. Now we may use the above to prove the following result of Kishimoto and Takai ([9, Theorem 2]).

Theorem 3.2.2. Let $\beta: G \rightarrow A u t B$ be a (strongly continuous) action of a compact abelian group $G$ with large spectral subspaces, then the $C^{*}$-algebra $B^{\beta} \otimes \mathscr{K} \otimes$ $\mathscr{K}\left(L^{2}(G)\right)$ is isomorphic to $(B \otimes \mathscr{K}) \times_{\beta \otimes \imath} G$.

Proof. We write $\bar{\beta}=\beta \otimes$ c for the product action of $G$ on $B \otimes \mathscr{K}$. It is easy to see that $B^{\beta} \otimes \mathscr{K} \otimes \mathscr{K}\left(L^{2}(G)\right)=(B \otimes \mathscr{K})^{\bar{\beta}} \otimes \mathscr{K}\left(L^{2}(G)\right) . \quad[9$, Lemma 4.7] gives the existence of unitaries $v_{\tau} \in M(B \otimes \mathscr{K})^{\bar{\beta}}(\tau), \tau \in \widehat{G}$ such that

$$
(B \otimes \mathscr{K})^{\bar{\beta}}(\tau)=(B \otimes \mathscr{K})^{\bar{\beta}} v_{\tau}
$$

(In the next section, we shall find $v_{1}$ explicitly for $B=\mathscr{O}_{A}$, and we can then take $v_{n}=v_{1}^{n}$, thus we do not actually use Kishimoto and Takai's lemma). Given the unitaries $v_{\tau}$, we may now describe the map which implements the isomorphism of $(B \otimes \mathscr{K})^{\bar{\beta}} \otimes \mathscr{K}\left(L^{2}(G)\right)$ with $(B \otimes \mathscr{K}) \times_{\bar{\beta}} G$ in two stages:

Firstly, for each $\chi$ and all $\gamma \in \widehat{G}, b \in B^{\beta}, i, j \in \mathbb{N}$ we have an isomorphism

$$
m_{\chi}:(B \otimes \mathscr{K})^{\bar{\beta}} \otimes M_{\chi} \lambda\left(C^{*}(G)\right) \rightarrow(B \otimes \mathscr{K})^{\bar{\beta}}(\bar{\chi}) \otimes \mathscr{K}\left(L^{2}(G)\right)^{A d \rho}(\chi)
$$

which is given by

$$
m_{\chi}\left(b \otimes e_{i j} \otimes M_{\chi} \lambda(\gamma)\right)=\left(v_{\chi \gamma}^{*}\left(b \otimes e_{i j}\right) v_{\gamma}\right) \otimes M_{\chi} \lambda(\gamma)
$$

For each $\tau \in \widehat{G}, \lambda(\tau)$ is the rank one projection in $\mathscr{K}\left(L^{2}(G)\right)$ onto the subspace spanned by $\tau$, hence the series $\sum_{\tau \in \widehat{G}} v_{\tau} \otimes \lambda(\tau)$ converges strictly in $M(B$ $\left.\otimes \mathscr{K} \otimes \mathscr{K}\left(L^{2}(G)\right)\right)$ to a unitary $V$. Conjugating by $V$ gives an isomorphism

$m:(B \otimes \mathscr{K})^{\bar{\beta}} \otimes \mathscr{K}\left(L^{2}(G)\right) \rightarrow \overline{\operatorname{span}}\left\{(B \otimes \mathscr{K})^{\bar{\beta}}(\bar{\chi}) \otimes \mathscr{K}\left(L^{2}(G)\right)^{A d \rho}(\chi): \chi \in \bar{G}\right\}$

which restricts to $m_{\chi}$ on $(B \otimes \mathscr{K})^{\bar{\beta}} \otimes M_{\chi} \lambda\left(C^{*}(G)\right)$. By [9, Proposition 3.1], the closed span on the right hand side of $(10)$ is precisely $\left(B \otimes \mathscr{K} \otimes \mathscr{K}\left(L^{2}\right.\right.$ $(G)))^{\bar{\beta}^{\otimes} A d \rho}$.

Secondly, we note that if the action $\beta$ on $B$ has large spectral subspaces, then so does the action $\bar{\beta}$ on $B \otimes \mathscr{K}$, thus we may apply the previous lemma to give us an isomorphism of $\left(B \otimes \mathscr{K} \otimes \mathscr{K}\left(L^{2}(G)\right)\right)^{\bar{\beta} \otimes A d \rho}$. with $(B \otimes \mathscr{K}) \times_{\bar{\beta}} G$. Under this isomorphism we see that 


$$
\left.\left(v_{\chi \gamma}^{*}\left(b \otimes e_{i j}\right) v_{\gamma}\right) \otimes M_{\chi} \lambda(\gamma) \mapsto i_{B \otimes \mathscr{K}}\left(v_{\chi \gamma}^{*}\left(b \otimes e_{i j}\right) v_{\gamma}\right)\right) i_{G}(\gamma)
$$

where by definition, $i_{G}(\gamma)$ is the extension of $i_{G}: G \rightarrow U M(B \otimes \mathscr{K})$ to $\widehat{G} \subset C(\widehat{G})$ $\subset L^{1}(G)$, which completes the proof.

\subsection{Construction of $\boldsymbol{v}_{1}$ for $\mathscr{O}_{A}$}

We now specialise to the case of the guage action on the Cuntz Krieger algebra $\mathscr{O}_{A}$ of a row finite matrix $A$ satisfying $(\mathrm{J})$. Here we construct a unitary operator $v_{1} \in M\left(\mathscr{O}_{A} \otimes \mathscr{K}\right)^{\bar{\alpha}}(1)$ which implements the 1-grading of the algebra $\mathscr{O}_{A} \otimes \mathscr{K}$; i.e. $\quad\left(\mathscr{O}_{A} \otimes \mathscr{K}\right)^{\bar{\alpha}}(1)=\left(\mathscr{O}_{A} \otimes \mathscr{K}\right)^{\bar{\alpha}} v_{1}$ where we again write $\bar{\alpha}$ for the product action $\alpha \otimes$ c of $\mathrm{T}$ on $\mathscr{O}_{A} \otimes \mathscr{K}$.

Before we start, we give some background details, which may be found in [2] that will be used frequently throughout the construction. For a $C^{*}$-algebra $B$, the strict topology of $M(B)$ is generated by the seminorms $\lambda_{b}(x)=\|b x\|$ and $\rho_{b}(x)=\|x b\|$ for each $b \in B$ and $x \in M(B)$. Since $M(B)$ is complete in the strict topology, a routine $\frac{\epsilon}{3}$ argument gives the following lemma:

Lemma 3.3.1. Let $\mathscr{B}$ be a dense subset of $B$, and $\left\{w_{n}\right\}$ a norm-bounded sequence in $M(B)$ such that $\left\{w_{n} b\right\}$ and $\left\{b w_{n}\right\}$ are Cauchy sequences for all $b \in \mathscr{B}$. Then $\left\{w_{n}\right\}$ converges strictly in $M(B)$.

Now we carry out the contruction of $v_{1}$. First notice that for each $i \in \mathbf{N}$ the operators $S_{i} \otimes e_{1 i}$ are partial isometries with mutually orthogonal initial spaces $\mathscr{H} \otimes e_{i}$ and mutually orthogonal range spaces $\left(S_{i} \mathscr{H}\right) \otimes e_{1}$. Hence the infinite sum $\sum_{i=1}^{\infty}\left(S_{i} \otimes e_{1 i}\right)$, converges strongly to an isometry $u$ of $\mathscr{H} \otimes \mathscr{H}=$ span $\left\{\mathscr{H} \otimes e_{i}\right\}$ onto $\mathscr{H} \otimes e_{1}$. We claim that this series in fact converges strictly in $M\left(\mathscr{O}_{A} \otimes \mathscr{K}\right)^{\bar{\alpha}}(1)$.

To apply 3.3.1, we first note that the partial sums $u_{n}=\sum_{i=1}^{n}\left(S_{i} \otimes e_{1 i}\right)$ are all partial isometries, and hence $\left\|u_{n}\right\|=1$ for all $n$. For the dense subalgebra required in the Lemma, we take $\mathscr{B}=\operatorname{span}\left\{S_{\mu} P_{r} S_{\nu}^{*} \otimes e_{j k}\right\}$, where $r_{,} j, k \in \mathbf{N}$, and $\mu, \nu \in \Sigma_{A}$ (which is dense in $\mathscr{O}_{A} \otimes \mathscr{K}$ since $A$ is row finite). For a given generator $c=S_{\mu} P_{r} S_{\nu}^{*} \otimes_{e}{ }_{j k}$, provided $n \geq j$ we have that

$$
\begin{aligned}
u_{n} c & =\left(\sum_{i=1}^{n} S_{i} \otimes e_{1 i}\right)\left(S_{\mu} P_{r} S_{\nu}^{*} \otimes e_{j k}\right) \\
& = \begin{cases}\left(S_{j} \otimes e_{1 j}\right)\left(S_{\mu} P_{r} S_{\nu}^{*} \otimes e_{j k}\right) & \text { if }|\mu| \geq 1 \text { and } A\left(\mu_{1}, j\right) \neq 0, \\
\left(S_{j} \otimes e_{1 j}\right)\left(P_{r} S_{\nu}^{*} \otimes e_{j k}\right) & \text { if }|\mu|=0 \text { and } A(r, j) \neq 0, \\
0 & \text { otherwise. }\end{cases} \\
& = \begin{cases}\left(S_{j} \otimes e_{1 j}\right) c & \text { or } \\
0 . & \end{cases}
\end{aligned}
$$


It follows that for any $b$ in the dense subalgebra $\mathscr{B}=\operatorname{span}\left\{S_{\mu} P_{r} S_{\nu} \otimes e_{j k}\right\}$, the sequence $\left\{u_{n} b\right\}$ is eventually constant and hence trivially convergent. Similarly

$$
\begin{aligned}
c u_{n} & =\left(S_{\mu} P_{r} S_{\nu}^{*} \otimes e_{j k}\right)\left(\sum_{i=1}^{n} S_{\imath} \otimes e_{1 i}\right) \\
& = \begin{cases}0 & \text { if } k \neq 1, \\
\left(S_{\mu} P_{r} S_{\nu|\nu|}^{*} \cdots S_{\nu_{2}}^{*} \otimes e_{j \nu|\nu|}\right) & \text { if }|\nu| \geq 0, \mid \nu 1=i \text { and } k=1, \\
\left(S_{\mu} S_{r} \otimes e_{\jmath r}\right) & \text { if }|\nu|=0 \text { and } k=1, i=r,\end{cases}
\end{aligned}
$$

which is constant if $n \geq \nu_{1}$ (or $n \geq r$ if $|\nu|=0$ ), and so by Lemma 3.3.1 the sequence $\left\{u_{n}\right\}$ converges strictly to $u \in M\left(\mathscr{O}_{A} \otimes \mathscr{K}\right)^{\bar{\alpha}}(1)$.

Because the summands are mutually orthogonal projections, the expansions

$$
\begin{aligned}
& u^{*} u=\sum_{i, j=1}^{\infty}\left(S_{j}^{*} S_{\imath} \otimes e_{j 1} e_{1 \imath}\right)=\sum_{i=1}^{\infty}\left(S_{\imath}^{*} S_{i} \otimes e_{\imath \imath}\right) \\
& u u^{*}=\sum_{i, j=1}^{\infty}\left(S_{\imath} S_{j}^{*} \otimes e_{1 i} e_{j 1}\right)=\sum_{\imath=1}^{\infty}\left(S_{\imath} S_{i}^{*} \otimes e_{11}\right),
\end{aligned}
$$

certainly converge strongly, and applications of Lemma 3.3.1 like that in the previous paragraph show that these too converge strictly in $M\left(\mathscr{O}_{A}^{\alpha} \otimes \mathscr{K}\right)$.

Following the construction in $[9, \S 4]$, we tensor with another copy of $\mathscr{K}$, which allows us the freedom to find isometries $v, w \in M\left(\mathscr{O}_{A}^{\alpha} \otimes \mathscr{K} \otimes \mathscr{K}\right)$ such that $x=v^{*}\left(u \otimes 1_{\mathscr{H}}\right) w$ is unitary. The infinite sums which appear below all consist of partial isometries with mutually orthogonal initial and range spaces and hence all their partial sums have norm 1 . When we pre- or post-multiply by a generator $S_{\mu} P_{r} S_{\nu}^{*} \otimes e_{j k} \otimes e_{p q}$ in the dense subalgebra span $\left\{S_{\mu} P_{r} S_{\nu}^{*} \otimes\right.$ $\left.e_{j k} \otimes e_{p q}\right\}$ of $\mathscr{O}_{A}^{\alpha} \otimes \mathscr{K} \otimes \mathscr{K}$ these sums are eventually constant, so Lemma 3.3.1 implies that they converge strictly in $M\left(\mathscr{O}_{A}^{\alpha} \otimes \mathscr{K} \otimes \mathscr{K}\right)$.

Choose an isometry $v: \mathscr{H} \otimes \mathscr{H} \rightarrow e_{11}(\mathscr{H}) \otimes \mathscr{H}$, such that

$$
\begin{aligned}
& v\left(e_{11} \otimes e_{11}\right) v^{*}=e_{11} \otimes e_{11} . \\
& w=\sum_{i=1}^{\infty}\left(S_{\imath} S_{i}^{*} \otimes V_{\imath}\right) \in M\left(\mathcal{O}_{A}^{\alpha} \otimes \mathscr{K} \otimes \mathscr{K}\right),
\end{aligned}
$$

where we note that $S_{\imath} S_{\imath}^{*} \otimes V_{\imath}$ are non-zero on orthogonal subspaces of $\mathscr{H} \otimes \mathscr{H}$ $\otimes \mathscr{H}$ and so $\left\|\sum_{\imath} S_{\imath} S_{\imath}^{*} \otimes V_{\imath}\right\|=1$ for all finite partial sums. Hence the product 


$$
w^{*} w=\sum_{\imath, j=1}^{\infty}\left(S_{,} S_{j}^{*} S_{\imath} S_{\imath}^{*} \otimes V_{j}^{*} V_{\imath}\right)
$$

converges strictly, which gives us that

$$
w^{*} w=\sum_{i=1}^{\infty}\left(S_{i} S_{\imath}^{*} \otimes V_{\imath}^{*} V_{\imath}\right)=\sum_{\imath=1}^{\infty}\left(S_{\imath} S_{i}^{*} \otimes 1_{\mathscr{H}} \otimes 1_{\mathscr{H}}\right)=1_{\mathscr{H}} \otimes 1_{\mathscr{H}} \otimes 1_{\mathscr{H}}
$$

since the $V_{\imath}$ are isometries. We also have that

$$
w w^{*}=\sum_{i, j=1}^{\infty}\left(S_{\imath} S_{\imath}^{*} S_{j} S_{j}^{*} \otimes V_{\imath} V_{\jmath}^{*}\right)=\sum_{i=1}^{\infty}\left(S_{\imath} S_{i}^{*} \otimes V_{\imath} V_{\imath}^{*}\right),
$$

this becomes,

$$
\left.\sum_{i=1}^{\infty}\left(S_{\imath} S_{\imath}^{*} \otimes\left(\sum_{j=1}^{\infty} A(j, i) e_{\jmath}\right) \otimes 1_{\mathscr{H}}\right)=\left(\sum_{j=1}^{\infty}\left(\sum_{\imath=1}^{\infty} A(j, i) S_{\imath} S_{i}^{*}\right) \otimes e_{\jmath \jmath}\right) \otimes 1_{\mathscr{H}}\right)
$$

finally, applying the Cuntz-Krieger relation (2) gives us that

$$
w w^{*}=\sum_{j=1}^{\infty}\left(S_{j}^{*} S_{\jmath} \otimes e_{\jmath \jmath} \otimes 1_{\mathscr{H}}\right)
$$

Thus we have shown that $w$ is an isometry of $\mathscr{H} \otimes \mathscr{H} \otimes \mathscr{H}$, onto the initial space of $u \otimes 1_{\mathscr{H}}$, and thus

$$
v_{1}=\left(1_{\mathscr{H}} \otimes v^{*}\right)\left(u \otimes 1_{\mathscr{H}}\right) w
$$

is a unitary in $M\left(\mathscr{O}_{A} \otimes \mathscr{K} \otimes \mathscr{K}\right)^{\bar{\alpha}}(1)$, where $\overline{\bar{\alpha}}=\alpha \otimes \iota \otimes_{\iota}$. As in [9, 4.7], we could identify $\left(\mathscr{O}_{A} \otimes \mathscr{K} \otimes \mathscr{K}, \mathbb{T}, \bar{\alpha}\right)$ with $\left(\mathscr{O}_{A} \otimes \mathcal{K}, \mathbb{T}, \bar{\alpha}\right)$, to get the required unitary $v_{1}$, we shall replace $\mathscr{K}$ by $\mathscr{K} \otimes \mathscr{K}$ and use (13) at the crucial steps.

\section{\$4. Computing the $\mathbb{K}-\mathbf{T}$ heory}

\subsection{The Dual Pimsner-Voiculescu Sequence}

Consider the gauge action $\alpha$ of $\mathbb{T}$ on $\mathscr{O}_{A}$ given in 2.1.9. From $[1, \S 10.6]$ (see also $[11, \S 3])$, there is a dual Pimsner-Voiculescu exact sequence for this action; 


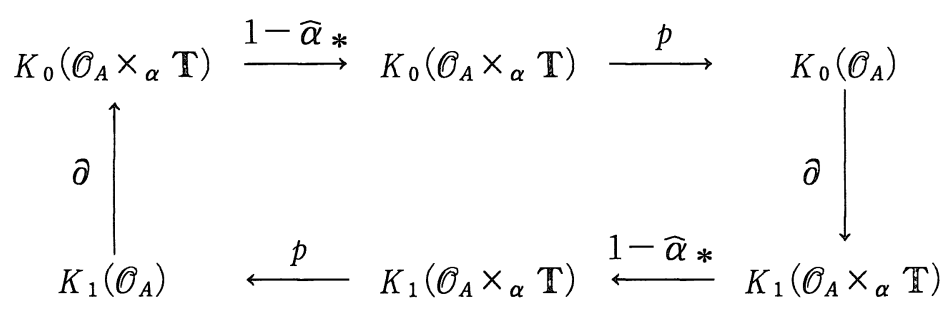

Figure 1.

where $\widehat{\alpha}$ is the homomorphism induced by the generator of the dual action of $\mathbb{Z}$ on $\mathscr{O}_{A} \times_{\alpha} \mathbb{T}$. We want to replace $\mathscr{O}_{A} \times_{\alpha} \mathbb{T}$ by the fixed point algebra $\mathscr{O}_{A}^{\alpha}$, so we need:

Lemma 4.1.1. The gauge action $\alpha$ on $\mathcal{O}_{A}$ has large spectral subspaces.

Proof. It suffices to check that $\overline{\mathcal{O}_{A}^{\alpha}(r){ }^{*} \mathfrak{O}_{A}^{\alpha}(r)}=\mathscr{O}_{A}^{\alpha}$ for each $r \in \mathbb{Z}$. We claim that $\mathcal{O}_{A}^{\alpha}(r) \supseteq \operatorname{span}\left\{S_{\mu} S_{\nu}^{*}:|\mu|-|\nu|=r\right\}$; this follows easily since $\alpha_{z}\left(S_{\mu}\right.$ $\left.S_{\nu}^{*}\right)=z^{|\mu|-|\nu|} S_{\mu} S_{\nu}^{*}$. Hence $\overline{\mathscr{O}_{A}^{\alpha}(r)^{*} \mathscr{O}_{A}^{\alpha}(r)}$ contains all norm limits of elements of the form $\left(S_{\mu} S_{\nu}^{*}\right) *\left(S_{\kappa} S_{\sigma}^{*}\right)$ where $|\mu|-|\nu|=|\kappa|-|\sigma|=\gamma$. Choosing $\mu=$ $\kappa$, and $\nu, \sigma$ freely in the above (note this implies that $|\nu|=|\sigma|$ ), we may thus construct any norm limit of $S_{\mu} S_{\nu}^{*},|\mu|=|\nu|$, whose span is dense in $\mathscr{O}_{A}^{\alpha}$.

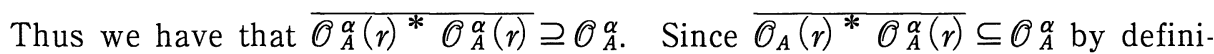
tion and the continuity of $\alpha$, this completes the proof.

Hence we may apply the results from the previous section to give us an isomorphism $\vartheta: \mathscr{O}_{A}^{\alpha} \otimes \mathscr{K} \otimes \mathscr{K}\left(L^{2}(\mathbb{T})\right) \rightarrow\left(\mathscr{O}_{A} \otimes \mathscr{K}\right) \times_{\bar{\alpha}} \mathbb{T}=\left(\mathscr{O}_{A} \times_{\alpha} \mathbb{T}\right) \otimes \mathscr{K} . \quad$ It is well known that for any rank one projection $e \in \mathscr{K}$, and any $C^{*}$-algebra $B$, the map $t: p \mapsto p \otimes e$ induces an isomorphism $t_{*}: K_{0}(B) \rightarrow K_{0}(B \otimes \mathscr{K})$, independent of the choice of $e$. Since, from 2.2.3, we know that $\mathscr{O}_{A}^{\alpha}$ is an AF algebra, we thus have that

$$
K_{*}\left(\mathscr{O}_{A} \times_{\alpha} \mathbb{T}\right) \cong K_{*}\left(\mathscr{O}_{A}^{\alpha}\right)= \begin{cases}K_{0}\left(\mathscr{O}_{A}^{\alpha}\right) & \text { if } *=0 \\ 0 & \text { if } *=1\end{cases}
$$

Applying this to the exact sequence in Figure 1, we obtain: 


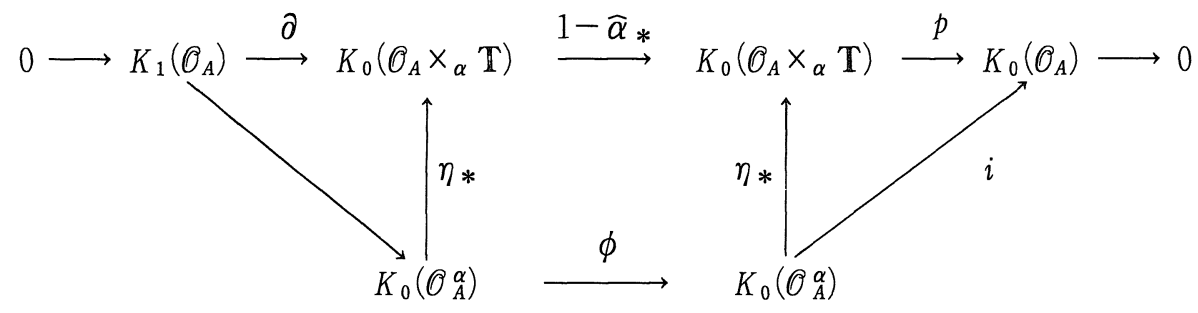

Figure 2.

where $\eta_{*}$ is the composition of the isomorphisms, $\vartheta_{*}, t_{*}$ mentioned above, $i$ is the homomorphism induced from commutativity of the right-hand triangle, and $\phi$ is induced to make the central square commute. From the lower exact sequence we may deduce that

$$
K_{0}\left(\mathscr{O}_{A}\right)=K_{0}\left(\mathscr{O}_{A}^{\alpha}\right) / \operatorname{Im} \phi \quad \text { and } \quad K_{1}\left(\mathscr{O}_{A}\right)=\operatorname{Ker} \phi
$$

In order to make any calcualtions, we need to know what $K_{0}\left(\mathscr{O}_{A}^{\alpha}\right), \phi$ and $i$ are.

Proposition 4.1.2. With notation as above, we have

$$
K_{0}\left(\mathscr{O}_{A}^{\alpha}\right)=\lim _{\longrightarrow}\left(\widetilde{\mathbb{Z}}^{\infty}, A^{t}\right)
$$

where $\widetilde{\mathbb{Z}}^{\infty}=\Perp_{i=1}^{\infty} \mathbb{Z}$, the additive group of all infinite sequences with integer coefficients which are eventually zero.

Proof. Recall from 2.2 .2 and 2.2 .3 that $\mathscr{O}_{A}^{\alpha}=\mathscr{F}_{A}$, is the direct limit $\overline{U_{k} \mathscr{F}_{k}}$ of a sequence of algebras, each of which is the countable direct sum $\mathscr{F}_{k}=\bigoplus i \mathscr{F}_{k}(i)$ of algebras isomorphic to $\mathscr{K}(\mathscr{H})$. Since $K_{0}(\mathscr{K})$ is generated by any minimal projection, to get a set of generators for $K_{0}\left(\mathscr{F}_{k}\right)$ it suffices to write down a minimal projection in each $\mathscr{F}_{k}(i)$. For this, choose any path $\mu(i)$ of length $k$ with $A\left(\mu_{k}(i), i\right)=1$, and take $\left[S_{\mu(i)} P_{i} S_{\mu(i)}^{*}\right]$. Thus, the map $\theta$ given by

$$
\left\{n_{\imath}\right\}_{i \in \mathbf{N}} \mapsto \sum_{i \in \mathbf{N}} n_{i}\left[S_{\mu(i)} P_{i} S_{\mu(i)}^{*}\right]
$$

is an isomorphism of the infinite direct sum $\widetilde{\mathbb{Z}}^{\infty}$ onto $K_{0}\left(\mathscr{F}_{k}\right)$. If we can compute the inclusions $\varphi_{k *}: K_{0}\left(\mathscr{F}_{k}\right) \hookrightarrow K_{0}\left(\mathscr{F}_{k+1}\right)$, we can use continuity to get $K_{0}$ $\left(\mathscr{F}_{A}\right)$.

The embedding $\varphi_{k}: \mathscr{F}_{k} \hookrightarrow \mathscr{F}_{k+1}$ sends $S_{\mu} P_{i} S_{\mu}^{*}$ to 


$$
\sum_{\{j: A(i, j)=1\}} S_{\mu} S_{i} S_{j} S_{j}^{*} S_{i}^{*} S_{\mu}^{*}
$$

If we write $\widetilde{\mu}(i)$ for the path $\left(\mu_{1}, \cdots, \mu_{k}, i\right) \in \sum_{A}$, we have

$$
\begin{aligned}
{\left[S_{\mu(i)} P_{\imath} S_{\mu(i)}^{*}\right]_{K_{0}\left(\mathscr{F}_{k+1}\right)} } & =\left[\sum_{\{l \cdot A(i, l)=1\}} S_{\widetilde{\mu}(i)} P_{l} S_{\widetilde{\mu}(i)}^{*}\right] K_{0}\left(\mathscr{F}_{k+1}\right) \\
& =\sum_{l=1}^{\infty} A(i, l)\left[S_{\widetilde{\mu}(i)} P_{l} S_{\widetilde{\mu}(i)}^{*}\right]_{K_{0}\left(\mathscr{F}_{k+1}\right)}
\end{aligned}
$$

Thus

$$
\sum_{i=1}^{\infty} n_{\imath}\left[S_{\mu(i)} P_{i} S_{\mu(i)}^{*}\right]_{K 0\left(\mathscr{F}_{k}\right)} \mapsto \sum_{l=1}^{\infty}\left(\sum_{i=1}^{\infty} A(i, l) n_{\imath}\right)\left[S_{\widetilde{\mu}(i)} P_{l} S_{\widetilde{\mu}(i)}^{*}\right]_{K 0}\left(\mathscr{F}_{k+1}\right)
$$

and so we have a commuting square:

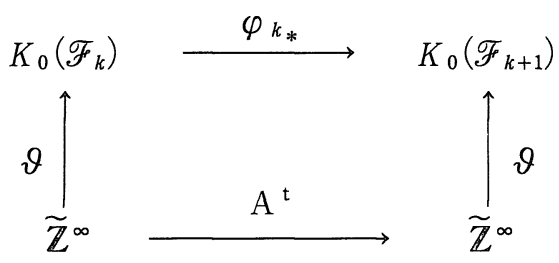

which gives the result.

\subsection{The Computation}

In order to proceed, we must calculate the effect of the map $\phi$ on $K_{0}\left(\mathcal{O}_{A}^{\alpha}\right)$ induced from $1-\widehat{\alpha} *$. To do this, we examine the central commuting square in Figure 2 above. In particular, we must calculate the effect of the isomorphisms comprising $\eta *$ on the generators of $K_{0}\left(\mathcal{O}_{A}^{\alpha}\right)$ as well as the dual action $1-\widehat{\alpha} *$. Expanding all the components comprising $\eta *$ we have: 


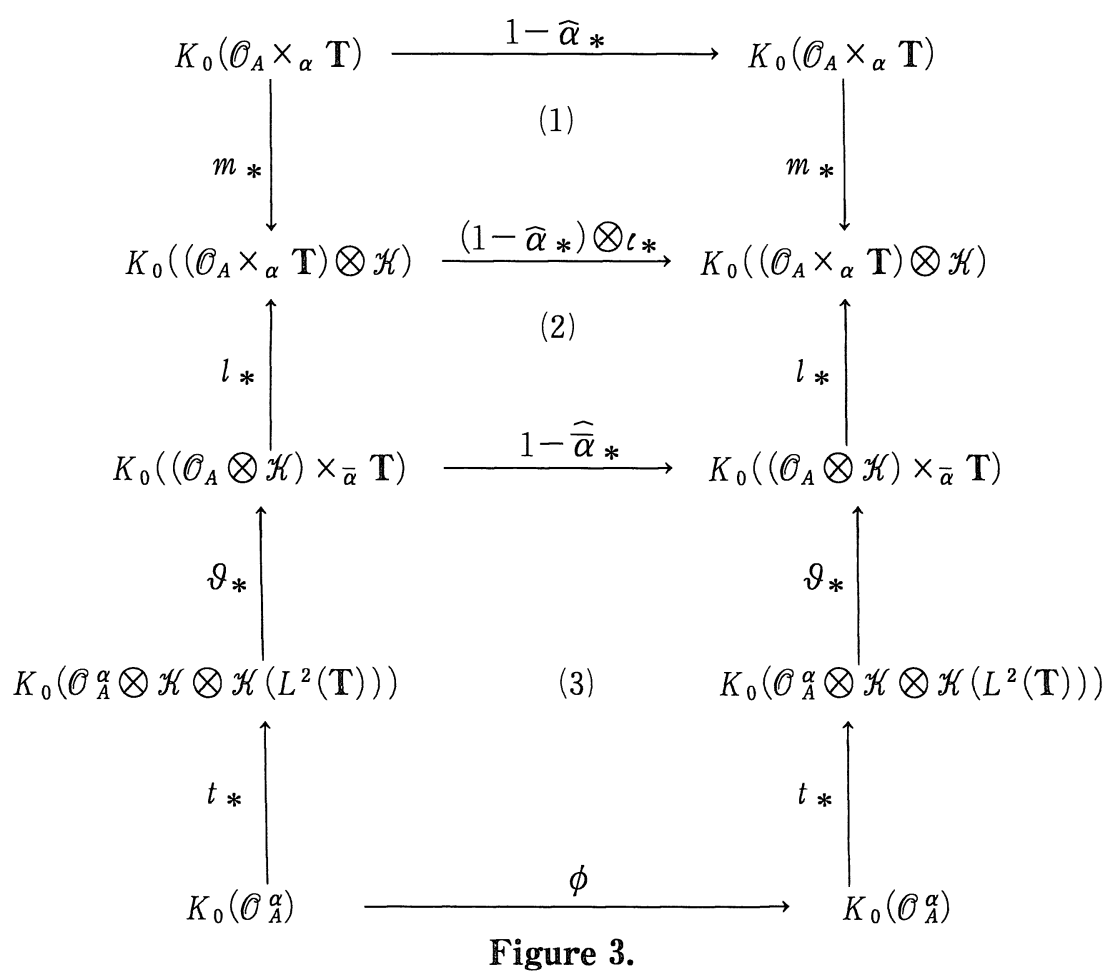

The maps $m_{*}, t_{*}$ are induced by the homomorphism $a \mapsto a \otimes e$ where $e$ is a rank one projection. The map $l_{*}$ is induced from the isomorphism of $\left(\mathscr{O}_{A} \otimes\right.$ $\mathscr{K}) \times_{\bar{\alpha}} \mathbf{T}$ and $\left(\mathscr{O}_{A} \times_{\alpha} \mathbf{T}\right) \otimes \mathscr{K}$, and the map $\vartheta *$ is described by equation (11). The square (1) in Figure 3 above commutes by the naturality of the maps, and square (2) commutes by the functoriality of $K_{0}$. Finally, the map $\phi$ is by definition the homomorphism which makes the square (3) commute.

Lemma 4.2.1. The map $\phi: K_{0}\left(\mathscr{O}_{A}^{\alpha}\right) \rightarrow K_{0}\left(\mathscr{O}_{A}^{\alpha}\right)$ induces the following commutative diagram:

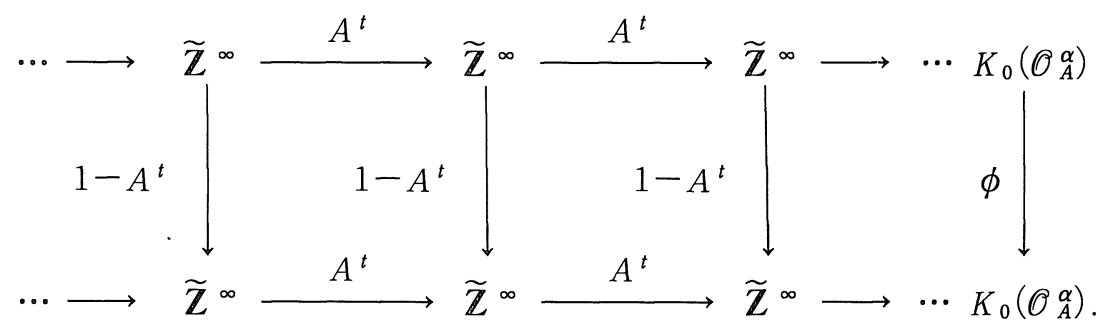


Proof. Since $K_{0}\left(\mathcal{O}_{A}^{\alpha}\right)=\lim _{\underline{0}} K_{0}\left(\mathscr{F}_{k}\right)=\underline{\lim }\left(\widetilde{\mathbb{Z}}^{\infty}, A^{t}\right)$, it is enough to compute $\phi$ on the image of $K_{0}\left(\mathscr{F}_{k}\right) \cong \widetilde{\mathbb{Z}}^{\infty}$. Under this isomorphism the generators are $\left[S_{\mu(i)} P_{i} S_{\mu(i)}^{*}\right]$, where $i \in \mathbb{N}$ and $\mu(i) \in \sum_{A}$ is any path of length $k$ ending at $i$. We write $\mu=\mu(i)$ and express $\phi\left(\left[S_{\mu} P_{i} S_{\mu}^{*}\right]\right)$ as a combination of classes of the same form. The isomorphism $t_{*}$ comes from tensoring by any rank-one projection $e \in \mathscr{K} \otimes \mathscr{K}\left(L^{2}(\mathbb{T})\right)$, and we can in particular choose $e=e_{11} \otimes \lambda\left(s^{q}\right)$, where $s^{q}$ denotes the function $s \mapsto s^{q}$ on $\mathbb{T}$. Thus we have that

$$
\left[t_{*}\left(S_{\mu} P_{i} S_{\mu}^{*}\right)\right]=\left[S_{\mu} P_{i} S_{\mu}^{*} \otimes e_{11} \otimes \lambda\left(s^{q}\right)\right]
$$

Next, we must examine the effect of the Kishimoto-Takai isomorphism $\vartheta_{*}$ on our element. From equations (9) and (11), we have

$$
\vartheta\left(S_{\mu} P_{i} S_{\mu}^{*} \otimes e_{11}\right) \otimes \lambda\left(s^{q}\right)=i_{\mathscr{O}_{A}} \otimes \mathcal{H}\left(v_{q}^{*}\left(S_{\mu} P_{i} S_{\mu}^{*} \otimes e_{11}\right) v_{q}\right) i_{\mathbb{T}}\left(s^{-q}\right),
$$

where

$$
\left(\mathscr{O}_{A} \otimes \mathscr{K}\right) \times_{\bar{\alpha}} \mathbb{T}=\overline{\operatorname{span}}\left\{i_{\mathscr{O}_{A} \otimes \mathcal{H}}(x) i_{\mathbb{T}}(z(s))\right\} \text { for } x \in \mathscr{O}_{A} \otimes \mathscr{K}, z(s) \in C(\mathbb{T}),
$$

as in 3.2.2, and $v_{q}=v_{1}^{q}$ is a unitary operator in $M\left(\mathscr{O}_{A} \otimes \mathscr{K}\right)^{\bar{\alpha}}(q)$. Taking $q=0$, in which case $v_{0}$ is the identity operator, we have that

$$
\begin{aligned}
& \vartheta_{*} \circ t_{*}\left(\left[S_{\mu} P_{i} S_{\mu}^{*}\right]\right)=\vartheta_{*}\left(\left[S_{\mu}^{*} P_{i} S_{\mu}^{*} \otimes e_{11} \otimes \lambda\left(s^{0}\right)\right]\right) \\
&=\left[i_{\mathscr{C}_{A}} \otimes \mathscr{H}\right. \\
&
\end{aligned}
$$

in $K_{0}\left(\left(\mathscr{O}_{A} \otimes \mathscr{K}\right) \times_{\bar{\alpha}} \mathbb{T}\right)$.

Next we calculate the effect of the dual action $\widehat{\bar{\alpha}} *$ on the class of our projection, and return to $1-\widehat{\bar{\alpha}}_{*}$ later. Since $\widehat{\bar{\alpha}}$ is given on $C_{C}(\mathbb{T})$ by $\widehat{\bar{\alpha}}(f(s))=$ $s f(s)$, we have $\widehat{\bar{\alpha}}\left(i_{\mathbb{T}}\left(s^{0}\right)\right)=i_{\mathbb{T}}\left(s^{1}\right)$, and so

$$
\widehat{\bar{\alpha}}_{*}\left(i_{\mathscr{O}_{A} \otimes \mathscr{K}}\left(S_{\mu} P_{i} S_{\mu}^{*} \otimes e_{11}\right) i_{\mathbb{T}}\left(s^{0}\right)\right)=i_{\mathscr{O}_{A} \otimes \mathcal{H}}\left(S_{\mu} P_{i} S_{\mu}^{*} \otimes e_{11}\right) i_{\mathbb{T}}\left(s^{1}\right) .
$$

In order to reverse the isomorphism $\vartheta_{*}$ on the right hand side of Figure 3 , we see from (14) that we must now conjugate $S_{\mu} P_{i} S_{\mu}^{*} \otimes e_{11}$ by $v_{-1}=v_{1}^{*}$. Thus we have that

$$
\left.\vartheta_{*}^{-1} \circ \overline{\bar{\alpha}}_{*} \circ t_{*}\left(\left[S_{\mu} P_{i} S_{\mu}^{*}\right]\right)=\left[v_{1}^{*}\left(S_{\mu} P_{i} S_{\mu}^{*} \otimes e_{11}\right) v_{1}\right) \otimes \lambda\left(s^{-1}\right)\right] .
$$

In order to apply the formula for $v_{1}$ from 3.3 , we expand $\mathscr{K}$ to $\mathscr{K} \otimes \mathscr{K}$ and put $v_{1}=\left(1 \otimes v^{*}\right)(u \otimes 1) w$. For $\mu=\left(\mu_{1}, \cdots, \mu_{k}\right)$, we have that 


$$
\begin{array}{r}
v_{1}^{*}\left(S_{\mu} P_{i} S_{\mu}^{*} \otimes e_{11} \otimes e_{11}\right) v_{1}=w^{*}\left(u^{*} \otimes 1\right)\left(S_{\mu} P_{i} S_{\mu}^{*} \otimes e_{11} \otimes e_{11}\right)(u \otimes 1) w \\
\text { by } \\
=w^{*}\left(S_{\mu_{2}} \cdots S_{\mu_{k}} P_{\imath} S_{\mu_{k}}^{*} \cdots S_{\mu_{2}}^{*} \otimes e_{\mu_{1} \mu_{1}} \otimes e_{11}\right) w \\
=\left(S_{\mu_{2}} S_{\mu_{2}}^{*} S_{\mu_{2}}\right) S_{\mu_{3}} \cdots S_{\mu_{k}} P_{i} S_{\mu_{k}}^{*} \cdots S_{\mu_{3}}^{*}\left(S_{\mu_{2}}^{*} S_{\mu_{2}} S_{\mu_{2}}^{*}\right) \otimes V_{\mu_{2}}^{*}\left(e_{\mu_{1} \mu_{1}} \otimes e_{11}\right) V_{\mu_{2}} .
\end{array}
$$

Since, $S_{\mu} \neq 0$ we have $A\left(\mu_{2}, \mu_{1}\right)=1$, and this becomes

$$
\left(S_{\mu_{2}} \cdots S_{\mu_{k}} P_{i} S_{\mu_{k}}^{*} \cdots S_{\mu_{2}}^{*}\right) \otimes e,
$$

where $e$ is some other rank 1 projection on $\mathscr{H} \otimes \mathscr{H}$. But this has the same class in $K_{0}\left(\mathscr{O}_{\mathrm{A}}^{\alpha} \otimes \mathcal{K} \otimes \mathscr{K}\right)$ as

$$
\left(S_{\mu_{2}} \cdots S_{\mu_{k}} P_{i} S_{\mu_{k}}^{*} \cdots S_{\mu_{2}}^{*}\right) \otimes e_{11} \otimes e_{11}
$$

Thus if we go back from $\left(\mathscr{K} \otimes \mathscr{K}, e_{11} \otimes e_{11}\right)$ to $\left(\mathscr{K}, e_{11}\right)$, we obtain

$$
\left[v_{1}^{*}\left(S_{\mu} P_{i} S_{\mu}^{*} \otimes e_{11}\right) v_{1}\right]=\left[S_{\mu_{2}} \cdots S_{\mu_{k}} P_{i} S_{\mu_{k}}^{*} \cdots S_{\mu_{2}}^{*} \otimes e_{11}\right]
$$

Hence,

$$
\vartheta_{*}^{-1} \circ \widehat{\bar{\alpha}} * \circ t_{*}\left(\left[S_{\mu} P_{\iota} S_{\mu}^{*}\right]\right)=\left[\left(v_{1}^{*}\left(S_{\mu} P_{\imath} S_{\mu}^{*} \otimes e_{11}\right) v_{1}\right) \otimes \lambda\left(s^{-1}\right)\right]
$$

which, in $K_{0}\left(\mathscr{O}_{A}^{\alpha} \otimes \mathscr{K} \otimes \mathscr{K}\left(L^{2}(\mathbf{T})\right)\right)$ is

$$
\left[\left(S_{\mu_{2}} \cdots S_{\mu_{k}} P_{i} S_{\mu_{k}}^{*} \cdots S_{\mu_{2}}^{*}\right) \otimes e_{11} \otimes \lambda\left(s^{-1}\right)\right]
$$

We note that the length of the path $\mu$ has been decreased by 1 ; to rewrite this in terms of projections in the original $\mathscr{F}_{k}$ we may use (cf. [3, p.32]) the infinite Cuntz-Krieger relation (2), to write it as

$$
\left[\left(\sum_{j=1}^{\infty} A(i, j) S_{\mu_{2}} \cdots S_{\mu_{k}} S_{\imath} P_{j} S_{i}^{*} S_{\mu_{k}}^{*} \cdots S_{\mu_{2}}^{*}\right) \otimes e_{11} \otimes \lambda\left(s^{-1}\right)\right]
$$

Provided $|\nu(j)|=k$, the class of the projection $S_{\nu(j)} P_{j} S_{\nu(j)}^{*}$ in $K_{0}\left(\mathscr{F}_{k}\right)$ is determined completely by $j$; thus $\vartheta_{*}^{-1} \circ\left(1-\widehat{\alpha}_{*}\right) \circ \vartheta_{*} \circ t_{*}\left(\left[S_{\mu(i)} P_{i} S_{\mu(i)}^{*}\right]\right)$ is given by

$$
\left[S_{\mu(i)} P_{\imath} S_{\mu(i)}^{*} \otimes e_{11} \otimes \lambda\left(s^{0}\right)\right]-\sum_{j=1}^{\infty} A(i, j)\left[S_{\nu(j)} P_{j} S_{\nu(j)}^{*} \otimes e_{11} \otimes \lambda\left(s^{-1}\right)\right]
$$


Finally, since the map $t_{*}$ is independent of the choice of projection, applying $t_{*}^{-1}$, gives

$$
\phi\left(\left[S_{\mu(\imath)} P_{\imath} S_{\mu(i)}^{*}\right]\right)=\left[S_{\mu(\imath)} P_{i} S_{\mu(i)}^{*}\right]-\sum_{j=1}^{\infty} A(i, j)\left[S_{\nu(j)} P, S_{\nu(j)}^{*}\right]
$$

Thus $\phi$ maps the image of $K_{0}\left(\mathscr{F}_{k}\right)$ in $K_{0}\left(\mathscr{O}_{A}^{\alpha}\right)$ into itself, and is given on $K_{0}$ $\left(\mathscr{F}_{k}\right) \cong \widetilde{\mathbb{Z}}^{\infty}$ by $1-A^{t}$. So we have a commuting diagram

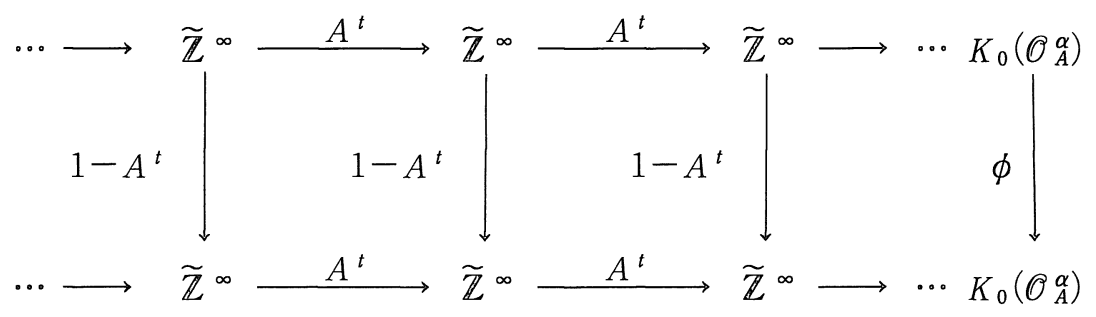

as required.

Lemma 4.2.2. The map $i$ in Figure 2 is the homomorphism induced by the inclusion $i_{A}: \mathscr{O}_{A}^{\alpha} \hookrightarrow \mathscr{O}_{A}$.

Proof. As in the previous result, we need only to check this on the generators of $K_{0}\left(\mathscr{O}_{A}^{\alpha}\right)$, namely $S_{\mu} P_{\imath} S_{\mu}^{*} \in K_{0}\left(\mathscr{F}_{k}\right)$, for $j \in \mathbb{N},|\mu|=k \geq 0$. We have already calculated in (15) that

$$
\vartheta_{*} \circ t_{*}\left(\left[S_{\mu} P_{\imath} S_{\mu}^{*}\right]\right)=\left[i_{\mathscr{O}_{A} \otimes \mathscr{H}}\left(S_{\mu} P_{i} S_{\mu}^{*} \otimes e_{11}\right) i_{\mathbb{T}}\left(s^{0}\right)\right]
$$

in $K_{0}\left(\left(\mathscr{O}_{A} \otimes \mathscr{K}\right) \times_{\bar{\alpha}} \mathbb{T}\right)$. Since $\bar{\alpha}=\alpha \otimes \iota, l *$ maps this into

$$
\left[\left(i_{\mathscr{O}_{A}}\left(S_{\mu} P_{i} S_{\mu}^{*}\right) i_{\mathbb{T}}\left(s^{0}\right)\right) \otimes e_{11}\right]
$$

Finally $m_{*}$ strips off the rank one projection $e_{11}$ hence $\eta *\left(\left[S_{\mu} P_{\imath} S_{\mu}^{*}\right]\right)$ is the class of $i_{\mathscr{O}_{A}}\left(S_{\mu} P_{\imath} S_{\mu}^{*}\right) i_{\mathbf{T}}\left(s^{0}\right)$ in $K_{0}\left(\mathscr{O}_{A} \times{ }_{\alpha} \mathbb{T}\right)$.

Next, the homomorphism $p$ shown in Figure 2 is induced from the embedding of $\mathscr{O}_{A} \times_{\alpha} \mathbb{T}$ in $\left(\mathscr{O}_{A} \times_{\alpha} \mathbb{T}\right) \times \bar{\alpha} \mathbb{Z}$, which gives the Takai isomorphism $\left(\mathscr{O}_{A}\right.$ $\left.\times_{\alpha} \mathbb{T}\right) \times_{\bar{\alpha}} \mathbb{Z} \cong \mathscr{O}_{A} \otimes \mathscr{K}$ and the identification of $K_{0}\left(\mathscr{O}_{A} \otimes \mathscr{K}\right.$ ) with $K_{0}\left(\mathscr{O}_{A}\right)$ (see $[14$, Theorem 6$])$. From $[14$, p.326], we see that the embedding is given by

$$
j_{\mathscr{O}_{A}} \times j_{\mathbb{T}}\left(i_{\mathscr{O}_{A}}\left(S_{\mu} P_{\imath} S_{\mu}^{*}\right) i_{\mathbb{T}}\left(s^{0}\right)\right)=j_{\mathscr{O}_{A}}\left(S_{\mu} P_{i} S_{\mu}^{*}\right) j_{\mathbb{T}}\left(s^{0}\right),
$$


and so

$$
p \circ \eta_{*}\left(\left[S_{\mu} P_{\imath} S_{\mu}^{*}\right]\right)=\left[\left(S_{\mu} P_{\imath} S_{\mu}^{*} \otimes 1\right)\left(1 \otimes \lambda\left(s^{0}\right)\right)\right]=\left[S_{\mu} P_{\imath} S_{\mu}^{*} \otimes f\right],
$$

where $f=\lambda\left(s^{0}\right)$ is a rank one projection in $\mathscr{K}\left(L^{2}(\mathbb{T})\right)$. The identification of $K_{0}\left(\mathscr{O}_{A} \otimes \mathscr{K}\right)$ with $K_{0}\left(\mathscr{O}_{A}\right)$ removes this projection and so $p \circ \eta *\left(\left[S_{\mu} P_{i} S_{\mu}^{*}\right]\right)$ is the class of $S_{\mu} P_{\imath} S_{\mu}^{*}$, viewed as a projection in $\mathscr{O}_{A}$ rather than $\mathscr{O}_{A}^{\alpha}$. This completes the proof of the lemma.

Corollary 4.2.3. $K_{0}\left(\mathscr{O}_{A}\right)$ is generated by the equivalence classes $\left\{\left[P_{t}\right]: i \in \mathbf{N}\right\}$.

Proof. From the previous lemma, and the exactness of the sequence given in Figure 2 we may deduce that the images of $\left[S_{\mu} P_{\imath} S_{\mu}^{*}\right]$ generate $K_{0}\left(\mathscr{O}_{A}\right)$. However, within $\mathscr{O}_{A}$ (though not in $\mathscr{O}_{A}^{\alpha}$ ) this projection is Murray-von Neumann equivalent to $\left[P_{1}\right]$, for each $i \in \mathbf{N}$. Thus, the map $i_{A *}$ is many to one, and sends the class of each $S_{\mu} P_{\imath} S_{\mu}^{*}$ which generate $K_{0}\left(\mathscr{O}_{A}^{\alpha}\right)$ to $\left[P_{i}\right]$ in $K_{0}\left(\mathscr{O}_{A}\right)$, which is the required result.

As in [3], we may represent the inductive limit $\lim _{(}\left(\widetilde{\mathbb{Z}}^{\infty}, A^{t}\right)$ as the set of equivalence classes of sequences $\left[\left\{x^{\jmath}\right\}_{j \in \mathbf{N}}\right]$, where $x^{\jmath} \in \widetilde{\mathbb{Z}}^{\infty}$ and $x^{j+1}=A^{t} x^{j}$ for $j$ greater than some $j_{0}$ where two sequences are identified if they differ only at a finite number of points. With this understanding, we have that $\phi\left(\left[\left\{x^{j}\right\}\right]\right)=$ $\left[\left\{x^{j}-A^{t} x^{j}\right\}\right]$, and again following [3, pp.32-33], we have:

Theorem 4.2.4. Let $A$ be a countably infinite 0-1 matrix which is row finite and satisfies condition $(\mathrm{J})$, then the map $\omega: \widetilde{\mathbb{Z}}^{\infty} \rightarrow \underline{\lim }\left(\widetilde{\mathbb{Z}}^{\infty}, A^{t}\right)$ given by $\omega(x)$ $=\left[\left\{\left(A^{t}\right)^{j} x\right\}\right]$ induces an isomorphism of $\widetilde{\mathbb{Z}}^{\infty} /\left(1-A^{t}\right) \widetilde{\mathbb{Z}}^{\infty}$ onto $\underline{\lim }\left(\widetilde{\mathbb{Z}}^{\infty}, A^{t}\right) / \operatorname{Im} \phi$ $\cong K_{0}\left(\mathscr{O}_{A}\right)$, and $\operatorname{Ker}\left\{\left(1-A^{t}\right): \widetilde{\mathbb{Z}}^{\infty} \rightarrow \widetilde{\mathbb{Z}}^{\infty}\right\}$ onto $\operatorname{Ker} \phi \cong K_{1}\left(\mathscr{O}_{A}\right)$.

Proof. For the first part, note that $\omega\left(\left(1-A^{t}\right) y\right) \in \operatorname{Im} \phi$ for all $y \in \widetilde{\mathbb{Z}}^{\infty}$, so the induced homomorphism is well defined. If $\left[\left\{x^{j}\right\}\right] \in \underline{\lim }\left(\widetilde{\mathbb{Z}}^{\infty}, A^{t}\right) / \operatorname{Im} \phi$, then we see that

$$
\begin{aligned}
{\left[\left\{x^{j}\right\}\right]+\operatorname{Im} \phi } & =\left[\left\{A^{t} x^{\jmath}\right\}\right]+\left[\left\{x^{\jmath}-A^{t} x^{\jmath}\right\}\right]+\operatorname{Im} \phi \\
& =\left[\left\{A^{t} x^{\jmath}\right\}\right]+\operatorname{Im} \phi .
\end{aligned}
$$

For sufficiently large $j_{0}$, we have that $\left\{x^{j}\right\}=\left\{x^{1}, x^{2}, \cdots, x^{\jmath_{0}}, A^{t} x^{\jmath_{0}},\left(A^{t}\right)^{2} x^{j_{0}}\right.$, $\cdots$,$\} . From j_{0}$ applications of $(16)$ we see that $\left[\left\{x^{j}\right\}\right]$ is equivalent, modulo $\operatorname{Im} \phi$ to 


$$
\begin{aligned}
{\left[\left\{\left(A^{t}\right)^{j_{0}} x^{j}\right\}\right] } & =\left[\left\{\left(A^{t}\right)^{j_{0}} x^{1},\left(A^{t}\right)^{j_{0}} x^{2}, \ldots,\left(A^{t}\right)^{j_{0}} x^{j_{0}},\left(A^{t}\right)^{j_{0}} A^{t} x^{j_{0}}, \ldots\right\}\right] \\
& =\left[\left\{x^{j_{0}},\left(A^{t}\right) x^{j_{0}}, \cdots,\left(A^{t}\right)^{j_{0}} x^{j_{0}},\left(A^{t}\right)^{j_{0+1}} x^{j_{0}}, \ldots\right\}\right] \\
& =\omega\left(x^{j_{0}}\right) .
\end{aligned}
$$

Thus we have shown that the homomorphism $\omega$ is surjective.

Now suppose that $\omega(x) \in \operatorname{Im} \phi$, that is, there exists $\left[\left\{x^{j}\right\}\right] \in \underline{\lim }\left(\widetilde{\mathbb{Z}}^{\infty}, A^{t}\right)$ such that $\left[\left\{\left(A^{t}\right)^{j} x\right\}\right]=\left[\left\{x^{j}-A^{t} x^{j}\right\}\right]$. Then, for large $k$, we have $\left(A^{t}\right)^{k} x=$ $x^{k}-A^{t} x^{k}$, and so

$$
\begin{aligned}
x & =x-\left(A^{t}\right)^{k} x+\left(A^{t}\right)^{k} x \\
& \left.=\left(1-A^{t}\right)\left(1+A^{t}+\cdots+A^{t}\right)^{k-1}\right) x+\left(1-A^{t}\right) x^{k}
\end{aligned}
$$

which belongs to $\left(1-A^{t}\right) \widetilde{\mathbb{Z}}^{\infty}$, which implies that the map induced from $\omega$ is injective, and completes the proof of the first part.

For the second part we note that the induced map is well-defined, since if $x$ $\in \operatorname{Ker}\left\{\left(1-A^{t}\right): \widetilde{\mathbb{Z}}^{\infty} \rightarrow \widetilde{\mathbb{Z}}^{\infty}\right\}$ then

$$
\begin{aligned}
\phi(\omega(x)) & =\phi\left(\left[\left\{\left(A^{t}\right)^{j} x\right\}\right]\right) \\
& =\left[\left\{\left(A^{t}\right)^{j} x-\left(A^{t}\right)^{j+1} x\right\}\right] \\
& =\left[\left\{\left(A^{t}\right)^{j}\left(1-A^{t}\right) x\right\}\right] \\
& =[\{0\}] .
\end{aligned}
$$

If $\phi\left(\left[\left\{x^{j}\right\}\right]\right)=0$, then $x^{j}=A^{t} x^{j}=x^{j+1}$ for $j \geq j_{0}$. Hence $\left\{x^{j}\right\}$ is equivalent to the constant sequence $\left\{x^{j_{0}}, x^{j_{0}}, \cdots\right\}$, which is in $\operatorname{Ker}\left\{\left(1-A^{t}\right): \widetilde{\mathbb{Z}}^{\infty} \rightarrow \widetilde{\mathbb{Z}}^{\infty}\right\}$. Thus the induced map of $\left(1-A^{t}\right) \widetilde{\mathbb{Z}}^{\infty}$ to $\operatorname{Ker} \phi$ is surjective. Finally we note that the induced map is faithful, since, if $\omega(x)=[\{0\}]$ then $\left(A^{t}\right)^{k} x=0$ for large $k$, which implies $x=0$ because $A^{t} x=x$.

In the case of $K_{0}$, our calculations actually say more:

Corollary 4.2.5. $K_{0}\left(\mathscr{O}_{A}\right)$ is generated, as an abelian group by the family $\left[S, S_{i}^{*}\right]$, subject only to the relations

$$
\left[S_{i} S_{i}^{*}\right]=\sum_{j=1}^{\infty} A(i, j)\left[S_{j} S_{j}^{*}\right]
$$

induced by the Cuntz-Krieger relation (2).

Proof. What we have actually proved above was that the diagram 


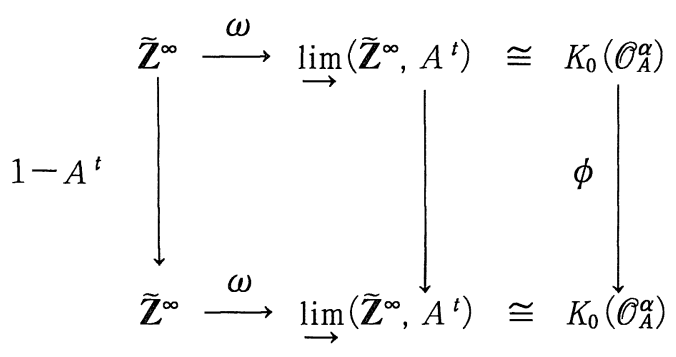

induces an isomorphism of $\widetilde{\mathbf{Z}}^{\infty} /\left(1-A^{t}\right) \widetilde{\mathbf{Z}}^{\infty}$ onto Coker $\phi \cong K_{0}\left(\mathscr{O}_{A}\right)$. Since the composition $\tilde{\mathbb{Z}}^{\infty} \rightarrow K_{0}\left(\mathscr{O}_{A}^{\alpha}\right) \rightarrow K_{0}\left(\mathscr{O}_{A}\right)$ is given by

$$
\left\{n_{i}\right\}_{i \in \mathrm{N}} \mapsto \sum_{i=1}^{\infty} n_{i}\left[S_{\mu(i)} P_{i} S_{\mu(i)}^{*}\right] \in K_{0}\left(\mathscr{F}_{k}\right) \mapsto \sum_{i=1}^{\infty} n_{i}\left[P_{i}\right] \in K_{0}\left(\mathscr{O}_{A}\right),
$$

this implies the result.

\section{\$5. Applications}

\subsection{Doplicher-Roberts Algebras}

The principal motivation for making the calculations given in this paper was to calculate the K-theory of the Doplicher-Roberts Algebras, $\mathscr{O}_{\rho}$ which are central to their nonabelian duality theory, [6],[7],[8]. These $C^{*}$-algebras are built from spaces of intertwiners between tensor powers of a given faithful representation $\rho: G \rightarrow S U(\mathscr{H})$, where $G$ is a compact group and $1<\operatorname{dim}(\mathscr{H})<\infty$. We refer to [10] for further details of their construction. Decomposing the tensor powers of $\rho$ into irreducible components yields a countable $0-1$ matrix $A_{\rho}$, which may be shown to be irreducible and row finite. From 2.1.12 $\mathscr{O}_{A_{\rho}}$ is simple, and so the map $\phi: \mathscr{O}_{\rho} \rightarrow \mathscr{O}_{A_{\rho}}$ given in [10, Theorem 2.1] is an isomorphism onto full corner of $\mathscr{O}_{A_{\rho}}$, and hence $\mathscr{O}_{\rho}$ is Morita equivalent to $\mathscr{O}_{A_{\rho}}$. Thus we have the following result:

Corollary 5.1.1. Let $\rho: G \rightarrow S U(\mathscr{H})$ be a faithful representation of a compact group, with $1<\operatorname{dim}(\mathscr{H})<\infty$, then

$$
K_{*}\left(\mathscr{O}_{\rho}\right) \cong K_{*}\left(\mathscr{O}_{A_{\rho}}\right) \cong \begin{cases}\operatorname{Coker}\left(1-A_{\rho}^{t}\right) & *=0 \\ \operatorname{Ker}\left(1-A_{\rho}^{t}\right) & *=1\end{cases}
$$


where $1-A_{\rho}^{t}$ is considered as a linear operator on $\widetilde{\mathbb{Z}}^{\infty}$.

We may identify $\widetilde{\mathbb{Z}}^{\infty}$ with the representation ring, $\mathscr{R}(G)$ of $G$ as follows: given a list of representatives of $\hat{G},\left\{\pi_{i}\right\}_{\imath \in \mathrm{N}}$ and the canonical basis $\left\{\varepsilon_{i}\right\}_{i \in \mathbb{N}}$ of $\widetilde{\mathbb{Z}}^{\infty}$, define a map $\Phi: \widetilde{\mathbb{Z}}^{\infty} \rightarrow \mathscr{R}(G)$ by $\xi_{i} \mapsto\left[\pi_{i}\right]$. It may be shown that $\Phi$ extends to an isomorphism of additive abelian groups, and that the map $A_{\rho}^{t}$ on $\widetilde{\mathbb{Z}}^{\infty}$ induces the map $\beta_{\rho}$ on $\mathscr{R}(G)$, where

$$
\beta_{\rho}:\left[\pi_{i}\right] \mapsto\left[\pi_{i} \otimes \rho\right]
$$

With this identification, we may restate the result 5.1.1 as:

Theorem 5.1.2. Let $\rho: G \rightarrow S U(\mathscr{H})$ be a faithful representation of a compact group, with $1<\operatorname{dim}(\mathscr{H})<\infty$, then

$$
K_{*}\left(\mathscr{O}_{o}\right) \cong \begin{cases}\operatorname{Coker}\left\{\left(1-\beta_{o}\right): \mathscr{R}(G) \rightarrow \mathscr{R}(G)\right\} & *=0 \\ \operatorname{Ker}\left\{\left(1-\beta_{\rho}\right): \mathscr{R}(G) \rightarrow \mathscr{R}(G)\right\} & *=1\end{cases}
$$

where $\beta_{\rho}$ is the linear operator on $\mathscr{R}(G)$ given by $\left[\pi_{i}\right] \mapsto\left[\pi_{i} \otimes \rho\right]$, for $i \in \mathbb{N}$.

\subsection{Examples}

Just for completeness, we calculate the $K$-groups of the infinite CuntzKrieger algebras $\mathscr{O}_{A_{1}}, \mathscr{O}_{A_{3}}$ we considered in Section 2 . Firstly we consider the linear operators $1-A_{1}^{t}$ and $1-A_{3}^{t}$ acting on $\widetilde{\mathbb{Z}}^{\infty}$ where

$$
1-A_{1}^{t}=\left(\begin{array}{rrrrr}
1 & -1 & 0 & 0 & \cdots \\
-1 & 0 & 0 & 0 & \cdots \\
0 & -1 & 0 & 0 & \cdots \\
0 & 0 & -1 & 0 & \cdots \\
\vdots & \vdots & \vdots & \vdots & \ddots
\end{array}\right) \quad 1-A_{3}^{t}=\left(\begin{array}{rrrrr}
1 & -1 & 0 & 0 & \cdots \\
-1 & 0 & -1 & 0 & \cdots \\
0 & -1 & 0 & -1 & \cdots \\
0 & 0 & -1 & 0 & \cdots \\
\vdots & \vdots & \vdots & \vdots & \ddots
\end{array}\right)
$$

It is easy to show that $\operatorname{Ker}\left(1-A_{1}^{t}\right)=\operatorname{Ker}\left(1-A_{3}^{t}\right)=\{0\}$ as operators on $\widetilde{\mathbb{Z}}^{\infty}$, even though $A_{3}^{t}$ has fixed points in the full infinite product of copies of $\mathbb{Z}$.

For $1-A_{1}^{t}$, we see that $y=\left(y_{1}, y_{2}, \cdots\right) \in \operatorname{Im}\left(1-A_{1}^{t}\right)$ provided $y_{1}+y_{2}=y_{3}$, in which case $\widetilde{\mathbb{Z}}^{\infty} / \operatorname{Im}\left(1-A_{1}^{t}\right) \cong \mathbb{Z}$. For $1-A_{3}^{t}$ we see that $y=\left(y_{1}, y_{2}, \ldots\right) \in \operatorname{Im}$ $\left(1-A_{3}^{t}\right)$ provided $\sum_{i=1}^{\infty}(-1)^{i}\left(y_{2 i}+y_{2 i-1}\right)=0$, in which case $\widetilde{\mathbb{Z}}^{\infty} / \operatorname{Im}\left(1-A_{3}^{t}\right) \cong \mathbb{Z}$ as well. Thus we have shown that

$$
\begin{array}{ll}
K_{0}\left(\mathscr{O}_{A_{1}}\right)=\mathbb{Z} & K_{1}\left(\mathscr{O}_{A_{1}}\right)=0 \\
K_{0}\left(\mathscr{O}_{A_{3}}\right)=\mathbb{Z} & K_{1}\left(\mathscr{O}_{A_{3}}\right)=0 .
\end{array}
$$




\section{References}

[1] Blackadar, B., K-Theory for Operatar Algebras, volume 5 of MSRI Publications. Springer, New York-Heidelberg-Berlin, 1986.

[2] Busby, R., Double centralizers and extensions of $C^{*}$-algebras, Trans. A.M.S., 132 (1968). 79-99.

[3] Cuntz, J., A class of $C^{*}$-algebras and toplogical Markov chains: Reducible chains and the Ext-functor for $C^{*}$-algebras, Invent. Math., 63(1981), 25-40.

[4] K-theory for certain $C^{*}$-algebras, Ann. Math., 113(1981), 181-197.

[5] Cuntz, J. and Krieger, W., A class of $C^{*}$-algebras and topological Markov chains, Invent. Math., 56 (1980), 251-268.

[6] Doplicher, S., and Roberts, J., Duals of compact Lie groups realized in the Cuntz algebras and their actions on $C^{*}$-algebras, J. Funct. Anal., 74(1987), 96-120.

[7] Compact group actions on $C^{*}$-algebras, J. Operator Theory, 19 (1988), 283-305.

[8] - Endomorphisms of $C^{*}$-algebras, cross products and duality for compact groups, Ann. Math., $\mathbf{1 3 0}$ (1989), 75-119.

[9] Kishimoto, A. and Takai, H., Some remarks on $C^{*}$-dynamical systems with a compact abelian group, Publ. RIMS Kyoto, 14(1978), 383-397.

[10] Mann, M., Raeburn, I., and Sutherland, C., Representations of finite groups and Cuntz-Krieger algebras, Bull. Aust. Math. Soc., 46 (1992), 225-243.

[11] Paschke, W., K-theory for actions of the circle group, J. Operator Theory, 6 (1981), 125-133.

[12] Pask, D. and Sutherland, C., Filtered inclusions of path algebras; a combinatorial approach to Doplicher-Roberts duality, J. Oper. Theory, 31 (1994), 99-121.

[13] Pedersen, G., $C^{*}$-Algebras and their Automorphism Groups, Academic Press, 1979.

[14] Raeburn, I., On crossed products and Takai duality, Proc. Edin. Math. Soc., 31 (1988), 321-330.

[15] Wagoner, J., Topological Markov chains $C^{*}$-algebras and $K_{2}, A b v$. Math., 71 (1988), 133-185. 
